\title{
Shrinkage Difference-Based Liu Estimators In Semiparametric Linear Models
}

\author{
Hadi Emami*and Sara Kiani \\ Department of Statistics, University of Zanjan, Iran.
}

\begin{abstract}
In this article, under a multicollinearity setting, we define difference-based Liu and non-Liu type shrinkage estimators along with their positive parts in the semiparametric linear model, when the errors are dependent and some nonstochastic linear restrictions are imposed. We derive the biases and exact risk expressions of these estimators and obtain the region of optimality of each estimator. Also, necessary and sufficient conditions, for the superiority of the difference-based Liu estimator over its counterpart, for choosing the Liu parameter $d$ are established. Finally, we illustrate the performance of these estimators with a simulation study.
\end{abstract}

Keywords Linear restriction, Multicollinearity, Preliminary test, Restricted Liu estimator, Risk function, Stein-type shrinkage

AMS 2010 subject classifications 62G08, 62J05, 62J07

DOI: $10.19139 /$ soic.v6i3.576

\section{Introduction}

Semiparametric linear models have received considerable attention in statistics and econometrics. Semiparametric models are by design more flexible than standard linear regression models since they combine both parametric and nonparametric components. In general the semiparametric linear model is defined by

$$
y=X \beta+f(t)+\epsilon
$$

where $y=\left(y_{1}, \ldots, y_{n}\right), X=\left(x_{1}, \ldots, x_{n}\right)$ is an $n \times p$ matrix, $f(t)=\left(f\left(t_{1}\right), \ldots, f\left(t_{n}\right)\right)$ and $\epsilon=\left(\epsilon_{1}, \ldots, \epsilon_{n}\right)$. We assume that in general, $\epsilon$ is a vector of disturbances, which is distributed as a multivariate normal, $N_{n}\left(0, \sigma^{2} V\right)$, where $V$ is a symmetric, positive definite known matrix and $\sigma^{2}$ is an unknown parameter. In general it is assumed that, $f($.$) is an unknown function, the t^{\prime} s$ have bounded support, say the unit interval, and have been reordered so that $t_{1} \leq t_{2} \leq \ldots \leq t_{n}$. Also the first derivative of $f($.$) is bounded by a constant, say L$. Most of approaches for Semiparametric linear models are based on different nonparametric regression procedures. There have been several methods to estimate $\beta$ and $f($.$) . Surveys regarding the estimation and application of the model (1) can be found in$ the monograph of Hardle et al.[8] and Muller [15].

In linear regression analysis one of the standard assumption is that all the explanatory variables are linearly independent. When this assumption is violated, the problem of multicollinearity enters into the data. The presence of multicollinearity may produce estimates with wrong signs or linear combination of the parameters may lead to wide confidence intervals for the individual parameters. Alternative estimators has been proposed to combat multicollinearity which are generally biased. Among them ridge estimator of Horel and Kennard [9] and Liu estimator (see, Liu [11],[12]) which combined the Stein [22] estimator with the ridge estimator have received a great deal of attention in statistical literature. It is well known that the incorporation of prior information available in

*Correspondence to: Hadi Emami (Email: h.emami@znu.ac.ir). Department of statistics, University of Zanjan, Iran.

ISSN 2310-5070 (online) ISSN 2311-004X (print)

Copyright (C) 2018 International Academic Press 
the form of restrictions provide better estimators than the ordinary estimator. However, when the prior information is doubtful, one may combine the restricted and ordinary estimators to obtain a better estimator, which leads to the preliminary tests and stein type shrinkage estimators. Recently these estimation techniques have been extended in semiparametr regression model (1). The approach of preliminary test estimation has been pioneered in Judge and Bock [10], Benda [5], Saleh [20], Saleh and Kibria [21] and very recently in Yuzbasi et al [27, 28] and Wu and Asr [25]. The main goal of shrinkage estimators is to develop necessary tools for computing the risk function of regression coefficient in a semiparametric linear model based on the eigenvalues of design matrix. Here we are looking for a new estimator for shrinkage parameter by making use of the existing ones in the literature. Our method is based on the differencing technique (see Yatchew [26] and Brown[6]). Compared to the estimation mentioned above, our method avoids the bandwidth choice. We consider difference-based shrinkage Liu estimators in comparison to the difference-based restricted Liu estimator. We give theoretical conditions that determine superiority among the estimation techniques in the mean squared error matrix sense. The paper is organized as follows. In Section 2, the model and the difference-based estimators are defined. We introduce difference-based shrinkage estimators in this section. In Section 3 bias and risk functions of the proposed estimators are obtained. In Section 4, the least/most values of the Liu parameter are identified for which the difference-based shrinkage Liu estimators dominate each other. Section 5 contains the simulation studies. Finally, some concluding results are stated in Section 6.

\section{The model and difference-based shrinkage estimators}

In this section, using the differencing technique we remove the nonparametric component in the semiparametric linear model and then propose some shrinkage estimates of the linear parameter in this model. The difference based technique has been used to by various authors. Let $\wp_{0}, \ldots, \wp_{m}$ be difference sequence satisfying

$$
\sum_{i=0}^{m} \wp_{i}=0, \quad \sum_{i=0}^{m} \wp_{i}^{2}=1,
$$

Now we define the $(n-m) \times n$ differencing matrix $D$ to have first and last row $\left(\wp^{\prime}, 0_{n-m-1}^{\prime}\right),\left(0_{n-m-1}^{\prime}, \wp^{\prime}\right)$ respectively, with $i$ th row $\left(0_{i}, \wp^{\prime}, 0_{n-m-i-1}^{\prime}\right) i=1, \ldots, n-m-1$, where 0 an $r$ vector of all zero elements.

$$
D=\left(\begin{array}{ccccccccc}
\wp_{0} & \wp_{1} & \wp_{2} & \ldots & \wp_{m} & 0 & \ldots & \ldots & 0 \\
0 & \wp_{0} & \wp_{1} & \wp_{2} & \ldots & \wp_{m} & 0 & \ldots & 0 \\
& & & & \vdots & & & \vdots & \\
0 & \ldots & \ldots & \wp_{0} & \wp_{1} & \wp_{2} & \ldots & \wp_{m} & 0 \\
0 & 0 & \ldots & \ldots & \wp_{0} & \wp_{1} & \wp_{2} & \ldots & \wp_{m}
\end{array}\right),
$$

Applying the differencing matrix to (1) we have

$$
\begin{aligned}
& D y=D X \beta+D f+D \epsilon \approx D X \beta+D \epsilon \\
& y_{D}=X_{D} \beta+\epsilon_{D}
\end{aligned}
$$

where $y_{D}=D y, X_{D}=D X, \epsilon_{D}=D \epsilon$ and $\operatorname{var}\left(\epsilon_{D}\right)=\sigma^{2} V_{D}=\sigma^{2} D V D^{\prime}$. Since $f$ is an unknown function with bounded first derivative, then $D f \approx 0$. To estimate $\beta$ in (2), we use the difference-based generalized least squares estimator (GLSE) given by

$$
\begin{gathered}
\hat{\beta}_{G}=C^{-1} X_{D}^{\prime} V_{D}^{-1} y_{D} \\
S^{2}=\frac{1}{n-p}\left(y_{D}-X_{D} \hat{\beta}_{G}\right)^{\prime} V_{D}^{-1}\left(y_{D}-X_{D} \hat{\beta}_{G}\right)
\end{gathered}
$$

where $C=X_{D}^{\prime} V_{D}^{-1} X_{D}$. Our primary interest is to estimate the linear parameters when it is a priori suspected but not certain that $\beta$ may be restricted to the subspace

$$
H \beta=h
$$


where $H$ is $q \times p$ non zero matrix with rank $q<p$ and $h$ is $q \times 1$ vector. The difference-based generalized restricted estimator $(G R E)$ of $\beta$ is given by

$$
\hat{\beta}_{G R}=\hat{\beta}_{G}+C^{-1} H^{\prime}\left(H C^{-1} H^{\prime}\right)^{-1}\left(h-H \hat{\beta}_{G}\right)
$$

In fact, the coefficient parameter $\beta$ can be regarded as a vector in $p$ dimensions space. If there exists multicollinearity in $X$ (or equivalently, $X_{D}$ is ill conditioned), the coefficients which is estimated by least square method, would be badly apart from the actual coefficient parameter in some directions of $p$ dimensions space, since the variance of $\hat{\beta}_{G R}$ depends on matrix $C$. In order to overcome the multicollinearity following Akdeniz et al [1], Swamy Mehta [23], Swamy et al [24], Roozbeh and Arashi [18] and Zhang and Yang [30] we minimize the sum of squared residuals with a spherical restriction $\left(\beta^{\top} \beta \leq \rho^{2}\right)$ and linear restriction. Therefore, a generalized optimization problem is given as follows:

$$
\begin{gathered}
\min \left(y_{D}-X_{D} \hat{\beta}_{G}\right)^{\prime} V_{D}^{-1}\left(y_{D}-X_{D} \hat{\beta}_{G}\right) \\
\operatorname{s.t}\left(d \hat{\beta}_{G}-\beta\right)^{\prime}\left(d \hat{\beta}_{G}-\beta\right) \leq \Phi^{2} \\
H \beta=h
\end{gathered}
$$

By using the Lagrangian method in relations (4), we can obtain the difference-based generalized restricted Liu estimation (GRLE)

$$
\hat{\beta}_{G R}(d)=\hat{\beta}_{G}(d)+C_{d}^{-1} H^{\prime}\left(H C_{d}^{-1} H^{\prime}\right)^{-1}\left(h-H \hat{\beta}_{G}(d)\right)
$$

where

$$
\hat{\beta}_{G}(d)=R_{d} \hat{\beta}_{G}, \quad R_{d}=C_{d}^{-1}(C+d I), \quad C_{d}=C+I
$$

$\hat{\beta}_{G}(d)$ is the difference-based generalized unrestricted Liu estimator (GULE) and $d \geq 0$ is the Liu parameter.

From Saleh [20], the likelihood ratio criterion for testing the null hypothesis $H \beta=h$, is given by

$$
£_{n}=\frac{\left(H \hat{\beta}_{G}-h\right)^{\prime}\left(H C^{-1} H^{\prime}\right)^{-1}\left(H \hat{\beta}_{G}-h\right)}{q S^{2}} .
$$

Under the null hypothesis and normal theory, $£_{n}$ follows a central F-distribution with $(q, n-p)$ degrees of freedom, while, under the alternative, it follows the non-central F-distribution with $(q, n-p)$ degrees of freedom and non-centrality parameter $\frac{1}{2} \Delta^{*}$, where

$$
\Delta^{*}=\frac{(H \hat{\beta}-h)^{\prime}\left(H C^{-1} H^{\prime}\right)^{-1}(H \hat{\beta}-h)}{\sigma^{2}} .
$$

In many practical situations, along with the model one may suspect that $\beta$ belongs to the subspace defined by (2.4). In such situation, as a result, we combine the difference based GULE and GRLE to obtain difference-based preliminary test generalized restricted Liu estimator (PTGRLE) as:

$$
\hat{\beta}_{G R}^{P T}(d)=\hat{\beta}_{G R}(d)+\left[1-I\left(£_{n} \leq F_{q, n-p}(\alpha)\right)\right]\left(\hat{\beta}_{G}(d)-\hat{\beta}_{G R}(d)\right)
$$

The difference-based PTGRLE has the disadvantage the it depends on $\alpha$, the level of significance, and also it yields the extreme results, namely $\hat{\beta}_{G R}(d)$ and $\hat{\beta}_{G}(d)$ depending on the outcome of the test. Later, we will discuss in detail of difference-based Stein-type generalized restricted Liu estimator (SGRLE) defined by

$$
\hat{\beta}_{G R}^{S}(d)=R_{d} \hat{\beta}_{G R}^{S}=\hat{\beta}_{G R}(d)+\left(1-v £_{n}^{-1}\right)\left(\hat{\beta_{G}}(d)-\hat{\beta}_{G R}(d)\right), \quad v=\frac{(q-2)(n-p)}{q(n-p+2)}, \quad q \geq 3
$$

where

$$
\hat{\beta}_{G R}^{S}=\hat{\beta}_{G R}+\left(1-v £_{n}^{-1}\right)\left(\hat{\beta}_{G}-\hat{\beta}_{G R}\right)
$$


is the difference-based Stein-type generalized restricted estimator (SGRE). Since the shrinkage factor $\left(1-d £_{n}^{-1}\right)$ becomes negative for $£<d$ and difference-based SGRLE has strange behavior for small values of $n$, so we define the difference-based positive-rule Stein-type generalized restricted Liu estimator (PRSGRLE) as

$$
\hat{\beta}_{G R}^{S+}(d)=R_{d} \hat{\beta}_{G R}^{S+}=\hat{\beta}_{G R}^{S}(d)-\left(1-v £_{n}^{-1}\right) I\left(£_{n} \leq v\right)\left(\hat{\beta}_{G}(d)-\hat{\beta}_{G R}(d)\right),
$$

where

$$
\hat{\beta}_{G R}^{S+}=\hat{\beta}_{G R}^{S}-\left(1-v £_{n}^{-1}\right) I\left(£_{n} \leq v\right)\left(\hat{\beta}_{G}-\hat{\beta}_{G R}\right),
$$

is the positive-rule Stein-type generalized restricted estimator (PRSGRE). The main objective of this study is to consider the performance of difference-based SGRLE and PRSGRLE. Recently the shrinkage estimation technique in various models has been considered by several researchers, for instance see, Arashi [2], Arashi et al [3], Roozbeh [17], Roozbeh and Arashi [4] and Norouzirad and Arashi [13] among others.

\section{Bias and Risk functions}

In this section, we provide the expressions for the bias and the quadratic risk of the estimators $\hat{\beta}_{G R}^{S}(d)$ and $\hat{\beta}_{G R}^{S+}(d)$.

\subsection{Biases of the estimators}

Here, we first present expressions for the biases of the difference-based SGRLE and difference-based PRSGRLE Theorem 3.1: Biases of the SGRLE and PRSGRLE, respectively, are given by:

$$
\begin{aligned}
b\left(\hat{\beta}_{G R}^{S}(d)\right) & =-(1-d) C_{d}^{-1} \beta-q v \delta R_{d} E\left[\chi_{q+2}^{-2}\left(\Delta^{*}\right)\right] \\
b\left(\hat{\beta}_{G R}^{S+}(d)\right) & =-(1-d) C_{d}^{-1} \beta-\frac{q v}{q+2} R_{d} \delta E\left[F_{q+2, n-p}^{-1}\left(\Delta^{*}\right)\right]-R_{d} \delta G_{q+2, n-p}\left(X^{\prime}, \Delta^{*}\right) \\
& +\frac{q v}{q+2} R_{d} \delta E\left[F_{q+2, n-p}^{-1}\left(\Delta^{*}\right) I\left(F_{q+2, n-p}\left(\Delta^{*}\right) \leq \frac{q v}{q+2}\right)\right]
\end{aligned}
$$

where

$$
\begin{gathered}
\left.G_{q+2 i, n-p}\left(X^{\prime}, \Delta^{*}\right)\right\}=\sum_{r=0}^{\infty} \frac{e^{\frac{-\Delta^{*}}{2}}\left(\frac{\Delta^{*}}{2}\right)^{r}}{\Gamma(r+1)} I_{x^{\prime}}\left[\frac{q+2 i}{2}+r, \frac{n-p}{2}\right] \\
E\left[\chi_{q+s}^{-2}\left(\Delta^{*}\right)\right]^{n}=\sum_{r=0}^{\infty} \frac{e^{\frac{-\Delta^{*}}{2}}\left(\frac{\Delta^{*}}{2}\right)^{r}}{\Gamma(r+1)} \frac{\Gamma\left(\frac{q+s}{2}+r-n\right)}{2^{n} \Gamma\left(\frac{q+s}{2}+r\right)} \\
E\left[F_{q+s, n-p}^{-j}\left(\Delta^{*}\right) I\left(F_{q+s, n-p}\left(\Delta^{*}\right)<\frac{q v}{q+s}\right)\right]= \\
\sum_{r=0}^{\infty} \frac{e^{\frac{-\Delta^{*}}{2}}\left(\frac{\Delta^{*}}{2}\right)^{r}}{\Gamma(r+1)}\left(\frac{q+s}{n-p}\right)^{j} \frac{B\left(\frac{q+s+2 r-2 j}{2}, \frac{n-p+2 j}{2}\right)}{B\left(\frac{q+s+2 r}{2}, \frac{n-p}{2}\right)} \times I_{X}\left(\frac{q+s+2 r-2 j}{2}, \frac{n-p+2 j}{2}\right),
\end{gathered}
$$

$X^{\prime}=\frac{q F_{\alpha}}{n-p+q F_{\alpha}}, X=\frac{q v}{n-p+q v}$ and $I_{X^{\prime}}$ is the Pearson regularized incomplete beta function,i.e.,

$$
I_{X^{\prime}}(a, b)=\frac{B_{x^{\prime}}(a, b)}{B(a, b)}=\frac{\int_{0}^{x^{\prime}} t^{a-1}(1-t)^{b-1}}{\int_{0}^{1} t^{a-1}(1-t)^{b-1}}
$$

Proof

We have

$$
\begin{aligned}
& b\left(\hat{\beta}_{G R}^{S}(d)\right)=E\left(\hat{\beta}_{G R}^{S}(d)-\beta\right)=E\left(\hat{\beta}(d)_{G}-\beta\right)-q v R_{d} \delta E\left[\chi_{q+2}^{-2}\left(\Delta^{*}\right)\right] \\
& -q v R_{d} \delta E\left[\chi_{q+2}^{-2}\left(\Delta^{*}\right)\right]-(1-d) C_{d}^{-1} \beta
\end{aligned}
$$




$$
\begin{aligned}
& b\left(\hat{\beta}_{G R}^{S+}(d)\right)=E\left(\hat{\beta}_{G R}^{S+}(d)-\beta\right)=E\left(\hat{\beta}_{G R}^{S}(d)-\beta\right)-R_{d} \delta G_{q+2, n-p}\left(X^{\prime}, \Delta^{*}\right) \\
& -\frac{q v}{q+2} R_{d} \delta E\left[F_{q+2, n-p}^{-1}\left(\Delta^{*}\right)\right]+\frac{q v}{q+2} R_{d} \delta E\left[F_{q+2, n-p}^{-1}\left(\Delta^{*}\right) I\left(F_{q+2, n-p}\left(\Delta^{*}\right) \leq \frac{q v}{q+2}\right)\right] \\
& =-(1-d) C_{d}^{-1} \beta-\frac{q v}{q+2} R_{d} \delta E\left[F_{q+2, n-p}^{-1}\left(\Delta^{*}\right)\right]-R_{d} \delta G_{q+2, n-p}\left(X^{\prime}, \Delta^{*}\right) \\
& +\frac{q v}{q+2} R_{d} \delta E\left[F_{q+2, n-p}^{-1}\left(\Delta^{*}\right) I\left(F_{q+2, n-p}\left(\Delta^{*}\right) \leq \frac{q v}{q+2}\right)\right] .
\end{aligned}
$$

\subsection{Risk of estimators}

In this subsection we will present the quadratic risk function. Suppose $\beta^{*}$ denotes an estimator of $\beta$, then for a given non-singular matrix $Q$, the loss function is defined as:

$$
L\left(\beta^{*} ; \beta\right)=\left(\beta^{*}-\beta\right)^{\prime} Q\left(\beta^{*}-\beta\right)
$$

and the corresponding risk function of the estimator $\beta^{*}$ is defined as:

$$
R\left(\beta^{*} ; \beta\right)=E\left(\beta^{*}-\beta\right)^{\prime} Q\left(\beta^{*}-\beta\right)=\operatorname{tr}(M) \text { for } Q=I_{p}
$$

where $M$ is the mean-square error matrix of the estimator $\beta^{*}$.

Theorem 3.2: Risks of SGRLE and PRSGRLE respectively are given by:

$$
\begin{aligned}
& R\left(\hat{\beta}_{G R}^{S}(d) ; \beta\right)= \sigma^{2} \operatorname{tr}\left(R_{d} C^{-1} R_{d}\right)+(1-d)^{2} \beta^{\prime} C_{d}^{-2} \beta+2 q v(1-d) \beta^{\prime} C_{d}^{-1} R_{d} \delta \\
& E\left(\chi_{q+2}^{-2}\left(\Delta^{*}\right)\right)-q v \sigma^{2} \operatorname{tr}\left(R_{d} C^{-1} H^{\prime}\left(H C^{-1} H^{\prime}\right)^{-1} H C^{-1} R_{d}\right)\{(q-2) \\
& E\left(\chi_{q+2}^{-4}\left(\Delta^{*}\right)\right)+\left[1-\frac{(q+2) \delta^{\prime} R_{d}^{\prime} R_{d} \delta}{2 \sigma^{2} \Delta^{*} \operatorname{tr}\left(R_{d} C^{-1} H^{\prime}\left(H C^{-1} H^{\prime}\right)^{-1} H C^{-1} R_{d}\right)}\right] \\
&\left.\left(2 \Delta^{*}\right) E\left(\chi_{q+4}^{-4}\left(\Delta^{*}\right)\right)\right\} \\
& R\left(\hat{\beta}_{G R}^{S+}(d) ; \beta\right)= R\left(\hat{\beta}_{G R}^{S}(d) ; \beta\right)-\sigma^{2} \operatorname{tr}\left(R_{d} C^{-1} H^{\prime}\left(H C^{-1} H^{\prime}\right)^{-1} H C^{-1} R_{d}\right) \\
& \times E\left[\left(1-\frac{q v}{q+2} F_{q+2, n-p}^{-1}\left(\Delta^{*}\right)\right)^{2} I\left(F_{q+2, n-p}\left(\Delta^{*}\right) \leq \frac{q v}{q+2}\right)\right] \\
&+ \delta^{\prime} R_{d}^{\prime} R_{d} \delta E\left[\left(1-\frac{q v}{q+4} F_{q+4, n-p}^{-1}\left(\Delta^{*}\right)\right)^{2} I\left(F_{q+4, n-p}\left(\Delta^{*}\right) \leq \frac{q v}{q+4}\right)\right] \\
&- 2 R_{d} \delta(1-d) \beta E\left[\left(\frac{q v}{q+2} F_{q+2, n-p}^{-1}\left(\Delta^{*}\right)-1\right)\right. \\
&\left.I\left(F_{q+2, n-p}\left(\Delta^{*}\right) \leq \frac{q v}{q+2}\right)\right]-2 \delta^{\prime} R_{d}^{\prime} R_{d} \delta E\left[\left(\frac{q v}{q+2} F_{q+2, n-p}^{-1}\left(\Delta^{*}\right)-1\right)\right. \\
&\left.I\left(F_{q+2, n-p}\left(\Delta^{*}\right) \leq \frac{q v}{q+2}\right)\right]
\end{aligned}
$$

\section{Proof}

Note that $\gamma=C^{\frac{-1}{2}} H^{\prime}\left(H C^{-1} H^{\prime}\right)^{-1} H C^{\frac{-1}{2}}$ is a symmetric idempotent matrix of rank $q \leq p$. Therefore, there exists an orthogonal matrix $\Gamma$, such that $\Gamma C^{\frac{-1}{2}} H^{\prime}\left(\left(H C^{-1} H^{\prime}\right)^{-1} H C^{\frac{-1}{2}}\right) \Gamma\left[\begin{array}{cc}I_{q} & 0 \\ 0 & 0\end{array}\right]$

$$
\begin{gathered}
\Gamma C^{\frac{-1}{2}} R_{d}^{\prime} R_{d} C^{\frac{-1}{2}} \Gamma=\left[\begin{array}{ll}
A_{11} & A_{12} \\
A_{21} & A_{22}
\end{array}\right]=A \\
A_{11}=\left(H C^{-1} H^{\prime}\right)^{-1} H C^{-1} R_{d}^{\prime} R_{d} C^{-1} H^{\prime}\left(H C^{-1} H^{\prime}\right)^{-1} \\
\eta_{1}^{\prime} A_{11} \eta_{1}=(H \beta-h)^{\prime}\left(H C^{-1} H^{\prime}\right)^{-1} H C^{-1} R_{d}^{\prime} R_{d} C^{-1} H^{\prime}\left(H C^{-1} H^{\prime}\right)^{-1}(H \beta-h) \\
=\delta^{\prime} R_{d}^{\prime} R_{d} \delta
\end{gathered}
$$


The matrices $A_{11}$ and $A_{22}$ are of order $q$ and $p-q$ respectively. Now, we define the random variable

$$
W=\Gamma C^{\frac{1}{2}} \hat{\beta}_{G}-\Gamma C^{\frac{-1}{2}} H^{\prime}\left(H C^{-1} H^{\prime}\right)^{-1} h
$$

So, $w \sim N_{q}\left(\eta, \sigma^{2} I_{p}\right)$ and $\eta=E(W)=\Gamma C^{\frac{1}{2}} \hat{\beta}-\Gamma C^{\frac{-1}{2}} H^{\prime}\left(H C^{-1} H^{\prime}\right)^{-1} h$. Partitioning the vectors $W=\left(w_{1}^{\prime}, w_{2}^{\prime}\right)^{\prime}$ and $\eta=\left(\eta_{1}^{\prime}, \eta_{2}\right)^{\prime}$, where are independent sub-vectors of order $q$ and $p-q$ respectively, $w_{1} \sim N_{q}\left(\eta_{1}, \sigma^{2} I_{p}\right)$ and $w_{2} \sim N_{p-q}\left(\eta_{2}, \sigma^{2} I_{p-q}\right)$, we obtain

$$
\begin{aligned}
\hat{\beta}_{G}-\beta & =C^{\frac{-1}{2}} \Gamma(w-\eta) \\
\hat{\beta}_{G}-\hat{\beta}_{G R} & =C^{-1} H^{\prime}\left(H C^{-1} H^{\prime}\right)^{-1}\left(H \hat{\beta}_{G}-h\right) \\
& =C^{-1} H^{\prime}\left(H C^{-1} H^{\prime}\right)^{-1} H C^{\frac{-1}{2}} \Gamma^{\prime} w
\end{aligned}
$$

Therefore, it follows that

$$
\begin{aligned}
\left(\hat{\beta}_{G}-\beta\right)^{\prime} R_{d}^{\prime} R_{d}\left(\hat{\beta}_{G}-\hat{\beta}_{G R}\right) & =(w-\eta)^{\prime} \Gamma C^{\frac{-1}{2}} R_{d}^{\prime} R_{d} C^{-1} H^{\prime}\left(H C^{-1} H^{\prime}\right)^{-1} H C^{\frac{-1}{2}} \Gamma^{\prime} w \\
& =(w-\eta)^{\prime} A \Gamma \gamma \Gamma^{\prime} w=(w-\eta)^{\prime}\left[\begin{array}{cc}
A_{11} & A_{12} \\
A_{21} & A_{22}
\end{array}\right]\left[\begin{array}{cc}
I_{q} & 0 \\
0 & 0
\end{array}\right] w
\end{aligned}
$$

Similarly,

$$
\left(\hat{\beta}_{G}-\hat{\beta}_{G R}\right)^{\prime} R_{d}^{\prime} R_{d}\left(\hat{\beta}_{G}-\hat{\beta}_{G R}\right)=w^{\prime}\left[\begin{array}{cc}
I_{q} & 0 \\
0 & 0
\end{array}\right]\left[\begin{array}{cc}
A_{11} & A_{12} \\
A_{21} & A_{22}
\end{array}\right]\left[\begin{array}{cc}
I_{q} & 0 \\
0 & 0
\end{array}\right] w^{\prime}=w_{1}^{\prime} A_{11} w_{1}
$$

Further, we conclude that

$$
£_{n}=\frac{w_{1}^{\prime} w_{1}}{q S^{2}} F_{q, n-p}\left(\Delta^{*}\right), \quad \Delta^{*}=\frac{(H \beta-h)\left(H C^{-1} H^{\prime}\right)^{-1}(H \beta-h)}{\sigma^{2}}
$$

Therefore, by making use of the [10] and (5) we can write:

$$
\begin{aligned}
R\left(\hat{\beta}_{G R}^{S} ; \beta\right) & =E\left[\left(R_{d} \hat{\beta}_{G R}^{S}-\beta\right)^{\prime}\left(R_{d} \hat{\beta}_{G R}^{S}-\beta\right)\right] \\
& =E\left[\left(R_{d} \hat{\beta}_{G R}-\beta\right)^{\prime}\left(R_{d} \hat{\beta}_{G R}-\beta\right)\right]+2 E\left[\left(R_{d} \hat{\beta}_{G R}-\beta\right)\left(1-v £_{n}^{-1}\right) \delta R_{d}\right] \\
& +E\left[\left(1-v £_{n}^{-1}\right) \delta R_{d}\right]^{2} \\
& =R\left(\hat{\beta}_{G}(d) ; \beta\right)-2 v E\left[£_{n}^{-1}\left(R_{d} \hat{\beta}_{G}-\beta\right)^{\prime} R_{d}\left(\hat{\beta}_{G}-\hat{\beta}_{G R}\right)\right] \\
& +v^{2} E\left[£^{-2}\left(\hat{\beta}_{G}-\hat{\beta}_{G R}\right) R_{d}^{2}\left(\hat{\beta}_{G}-\hat{\beta}_{G R}\right)\right] \\
& =R\left(\hat{\beta}_{G}(d) ; \beta\right)-2 v E\left[£_{n}^{-1}\left(w_{1}^{\prime} A_{11} W_{1}-\eta_{1}^{\prime} A_{11} w_{1}+w_{2}^{\prime} A_{21} W_{1}-\eta_{2}^{\prime} A_{21} w_{1}\right)\right] \\
& +v^{2} E\left[£_{n}^{-2}\left(w_{1}^{\prime} A_{11} W_{1}\right)\right]+2(1-d) v \delta^{\prime} R_{d} C_{d}^{-1} \beta E\left(\xi_{n}^{-1} w\right)
\end{aligned}
$$

But,

$$
\begin{aligned}
& E\left(\frac{q S^{2}}{w_{1}^{\prime} w_{1}}\right)=E\left[\left(\frac{q S^{2}}{w^{\prime} w}\right)\right]=E\left(\frac{q}{n-p} \frac{\chi_{n-p}^{2}}{\chi_{q}^{2}\left(\Delta^{*}\right)}\right)=q E\left[\chi_{q}^{-2}\left(\Delta^{*}\right)\right] \\
& E\left[\frac{q^{2} S^{4}}{\left(w^{\prime} w\right)^{2}}\right]=E\left(\frac{q^{2} S^{4}}{\left(w^{\prime} w\right)^{2}}\right)=\frac{q^{2}}{(n-p)}(n-p+2) E\left[\chi_{q}^{-4}\left(\Delta^{*}\right)\right]
\end{aligned}
$$

Therefore, gathering all terms we can obtain:

$$
\begin{aligned}
& R\left(\hat{\beta}_{G R}^{S}(d) ; \beta\right)=\sigma^{2} \operatorname{tr}\left(R_{d} C^{-1} R_{d}\right)+(1-d)^{2} \beta^{\prime} C_{d}^{-2} \beta+2 q v(1-d) \beta^{\prime} C_{d}^{-1} R_{d} \delta \\
& E\left(\chi_{q+2}^{-2}\right)-q v \operatorname{tr}\left(A_{11}\right) \sigma^{2}\left\{2 E\left[\chi_{q+2}^{-2}\left(\Delta^{*}\right)\right]+(q-2) E\left[\chi_{q+2}^{-4}\left(\Delta^{*}\right)\right]\right\} \\
& +(1-d)^{2} \beta^{\prime} C_{d}^{-2} \beta+\eta_{1}^{\prime} A_{11} \eta_{1} d q\left\{(q-2) E\left[\chi_{q+4}^{-4}\left(\Delta^{*}\right)\right]+2 E\left[\chi_{q+2}^{-2}\left(\Delta^{*}\right)\right]\right. \\
& \left.-2 E\left[\chi_{q+4}^{-2}\left(\Delta^{*}\right)\right]\right\}
\end{aligned}
$$


Now, we use the following identities to obtain the final from of the risk $\hat{\beta}_{G R}^{S}(d)$ given by (9),

$$
\begin{aligned}
& E\left[\chi_{p}^{-2}\left(\Delta^{*}\right)\right]-E\left[\chi_{p+2}^{-2}\left(\Delta^{*}\right)\right]=2 E\left[\chi_{p+2}^{-4}\left(\Delta^{*}\right)\right] \quad, p \geq 2 \\
& E\left[\chi_{p+2}^{-2}\left(\Delta^{*}\right)\right]-(p-2) E\left[\chi_{p+2}^{-4}\left(\Delta^{*}\right)\right]=\Delta^{*} E\left[\chi_{p+4}^{-4}\left(\Delta^{*}\right)\right] .
\end{aligned}
$$

By using (6), we have

$$
\begin{aligned}
R\left(\hat{\beta}_{G R}^{S+}(d) ; \beta\right) & =E\left[\left(\hat{\beta}_{G R}^{S+}(d)-\beta\right)^{\prime}\left(\hat{\beta}_{G R}^{S+}(d)-\beta\right)\right] \\
& =E\left[\left(R_{d} \hat{\beta}_{G R}^{S}-\beta\right)^{\prime}\left(R_{d} \hat{\beta}_{G R}^{S}-\beta\right)\right]-2 E\left[\left(R_{d} \hat{\beta}_{G R}^{S}-\beta\right)\left(1-v £_{n}^{-1}\right) I\left(£_{n} \leq v\right) R_{d}\left(\hat{\beta}_{G}-\hat{\beta}_{G R}\right)\right] \\
& +E\left[\left(1-v £_{n}^{-1}\right) I\left(£_{n} \leq v\right) R_{d}\left(\hat{\beta}_{G}-\hat{\beta}_{G R}\right)\right]^{2} .
\end{aligned}
$$

By, using (5)

$$
\begin{aligned}
& E\left[\left(R_{d} \hat{\beta}_{G R}-\beta\right)\left(1-v £_{n}^{-1}\right) I\left(£_{n} \leq v\right) R_{d}\left(\hat{\beta}_{G}-\hat{\beta}_{G R}\right)\right] \\
& =E\left[\left(R_{d} \hat{\beta}_{G R}\left(1-v £_{n}^{-1}\right) I\left(£_{n} \leq v\right) \delta R_{d}\right)-\left(\beta\left(1-v £_{n}^{-1}\right) I\left(£_{n} \leq v\right) \delta R_{d}\right)\right] \\
& =E\left[\left(R_{d}\left(\hat{\beta}_{G R}-\beta+\beta\right)\left(1-v £_{n}^{-1}\right) I\left(£_{n} \leq v\right) \delta R_{d}\right)-\left(\beta\left(1-v £_{n}^{-1}\right) I\left(£_{n} \leq v\right) \delta R_{d}\right)\right] \\
& =E\left\{\left(1-v £_{n}^{-1}\right) I\left(£_{n} \leq v\right)\left(\hat{\beta}_{G R}-\beta\right) R_{d}^{2}\left(\hat{\beta}_{G}-\hat{\beta}_{G R}\right)+\left[\left(1-v £_{n}^{-1}\right)^{2} I\left(£_{n} \leq v\right)\left(\hat{\beta}_{G}-\hat{\beta}_{G R}\right) R_{d}^{2}\left(\hat{\beta}_{G}-\hat{\beta}_{G R}\right)\right]\right. \\
& \left.+\left[\beta^{\prime}\left(R_{d}-I_{p}\right)\left(1-d £_{n}^{-1}\right) I\left(\xi_{n} \leq v\right) R_{d}\left(\hat{\beta}_{G}-\hat{\beta}_{G R}\right)\right]\right\} .
\end{aligned}
$$

Therefore, we get

$$
\begin{aligned}
R\left(\hat{\beta}_{G R}^{S+}(d) ; \beta\right) & =R\left(\hat{\beta}_{G R}^{S}(d) ; \beta\right)+E\left[\left(1-v £_{n}^{-1}\right)^{2} I\left(£_{n} \leq v\right)^{2}\left(\hat{\beta}_{G}-\hat{\beta}_{G R}\right) R_{d}^{2}\left(\hat{\beta}_{G}-\hat{\beta}_{G R}\right)\right] \\
& -2 E\left[\left(1-v £_{n}^{-1}\right) I\left(£_{n} \leq v\right)\left(\hat{\beta}_{G R}-\beta\right) R_{d}^{2}\left(\hat{\beta}_{G}-\hat{\beta}_{G R}\right)\right] \\
& -2 \beta^{\prime}\left(R_{d}-I_{d}\right) E\left[\left(1-v £_{n}^{-1}\right) I\left(£_{n} \leq v\right) R_{d}\left(\hat{\beta}_{G}-\hat{\beta}_{G R}\right)\right] \\
& =R\left(\hat{\beta}_{G R}^{S}(d) ; \beta\right)-\sigma^{2} \operatorname{tr}\left(R_{d} C^{-1} H^{\prime}\left(H C^{-1} H^{\prime}\right)^{-1} H C^{-1} R_{d}\right) \\
& \times E\left[\left(1-\frac{q v}{q+2} F_{q+2, n-p}^{-1}\left(\Delta^{*}\right)\right)^{2} I\left(F_{q+2, n-p}\left(\Delta^{*}\right) \leq \frac{q v}{q+2}\right)\right] \\
& +\delta^{\prime} R_{d}^{2} \delta E\left[\left(1-\frac{q v}{q+4} F_{q+4, n-p}^{-1}\left(\Delta^{*}\right)\right)^{2} I\left(F_{q+4, n-p}\left(\Delta^{*}\right) \leq \frac{q v}{q+4}\right)\right] \\
& -2(1-d) \delta^{\prime} R_{d} C_{d}^{-1} \beta E\left[\left(\frac{q v}{q+2} F_{q+2, n-p}^{-1}\left(\Delta^{*}\right)-1\right) I\left(F_{q+2, n-p}\left(\Delta^{*}\right) \leq \frac{q v}{q+2}\right)\right] \\
& -2 \delta^{\prime} R_{d}^{2} \delta E\left[\left(\frac{q v}{q+2} F_{q+2, n-p}^{-1}\left(\Delta^{*}\right)-1\right) I\left(F_{q+2, n-p}\left(\Delta^{*}\right) \leq \frac{q v}{q+2}\right)\right] .
\end{aligned}
$$

\section{Comparison of the estimators}

In this section, we compare the underlying estimators. We compare the shrinkage type estimators of $\beta$ based on the risk criterion as a function of the departure parameter. Comparisons needs the study of the derivatives of the Liu shrinkage estimators with respect to $d$. These procedures are adopted throughout in this section. Since $C$ is a positive definite matrix. Thus we can find an orthogonal matrix $\Gamma$ such that $C=\Gamma \Lambda \Gamma^{\prime}$ and $\Lambda=\Gamma^{\prime} C \Gamma=$ $\operatorname{Diag}\left(\lambda_{1}, \lambda_{2}, \ldots, \lambda_{p}\right)$ where

$$
\lambda_{1}>\lambda_{2}>\cdots>\lambda_{p} \geq 0
$$

are the eigenvalues of C. It is easy to see that the eigenvalues of $R_{d}=\left(C+I_{p}\right)^{-1}\left(C+d I_{p}\right)$ and $C_{d}=C+I$ are $\frac{\lambda_{i}+d}{\lambda_{i}+1}$ and $\lambda_{i}+1, i=1, \ldots, p$, respectively. With this background we get the following identities:

$$
\beta^{\prime} C_{d}^{-2} \beta=\beta^{\prime} \Gamma\left(\Lambda+I_{p}\right)^{-2} \Gamma^{\prime} \beta=\alpha^{\prime}\left(\Lambda+I_{p}\right)^{-2}=\sum_{i=1}^{p} \frac{\alpha_{i}^{2}}{\left(\lambda_{i}+1\right)^{2}} \quad \alpha=\Gamma^{\prime} \beta
$$




$$
\operatorname{tr}\left(R_{d} C^{-1} R_{d}\right)=\operatorname{tr}\left(C^{-1} R_{d}^{2}\right)=\sum_{i=1}^{p} \frac{\left(\lambda_{i}+d\right)^{2}}{\left(\lambda_{i}+1\right)^{2} \lambda_{i}}
$$

$$
\begin{aligned}
& \operatorname{tr}\left[R_{d} C^{-1} H^{\prime}\left(H C^{-1} H^{\prime}\right)^{-1} H C^{-1} R_{d}\right] \\
& =\operatorname{tr}\left[\Gamma(\Lambda+I)^{-1}(\Lambda+d) \Gamma C^{-1} H^{\prime}\left(H C^{-1} H^{\prime}\right)^{-1} H C^{-1} \Gamma(\Lambda+I)^{-1}(\Lambda+d) \Gamma C^{-1}\right] \\
& =\operatorname{tr}\left[\Gamma^{\prime} H^{\prime}\left(H C^{-1} H^{\prime}\right)^{-1} H \Gamma C^{-2}(\Lambda+I)^{-2}(\Lambda+d)^{2}\right] \\
& =\sum_{i=1}^{p} \frac{h_{i i}^{*}\left(\lambda_{i}+d\right)^{2}}{\lambda_{i}^{2}\left(\lambda_{i}+1\right)^{2}}
\end{aligned}
$$

where $h_{i i}^{*} \geq 0$ is the $i$ th diagonal element of $\Gamma^{\prime} H^{\prime}\left(H C^{-1} H^{\prime}\right)^{-1} H \Gamma=H^{*}$. Let us now compare the performance of the Liu shrinkage estimator with its usual counterpart. Comparison results concerning (GULE), (GRLE) and (PTGRLE) are well-known in the literature. Thus we focus on SGRLE and PRSGRLE in the sequel.

The SGRLE and PRSGRLE are superior to other proposed estimators in semiparametric linear models under following theorems:

Theorem 4.1.i. There always exist a positive $d \in\left(0, d_{1}^{*}\right)$ and $\left(0, d_{2}^{*}\right)$ such that SGRLE has smaller risk value than SGRE under $H_{0}: H \beta=h$ and $H_{A}: H \beta \neq h$, respectively, where

in which,

$$
\begin{aligned}
d_{1}^{*}= & \frac{\min _{1 \leq i \leq p}\left(v h_{i i}^{*} \lambda_{i}-1+\alpha_{i}^{2}\right)}{\max _{1 \leq i \leq p}\left(\frac{\alpha_{i}^{2}}{\sigma^{2}}+\frac{1}{\lambda_{i}}-v h_{i i}^{*}\right)} \\
d_{2}^{*}= & \frac{f_{1}\left(\alpha, \Delta^{*}\right)}{f_{2}\left(\alpha, \Delta^{*}\right)}
\end{aligned}
$$

$$
\begin{aligned}
f_{1}\left(\alpha, \Delta^{*}\right)= & \min _{1 \leq i \leq p}\left\{\sigma ^ { 2 } \left[\left(-1+q v(q-2) h_{i i}^{*} \lambda_{i} E\left(\chi_{q+2}^{-4}\left(\Delta^{*}\right)\right)\right)+2 q d \Delta^{*} h_{i i}^{*} \lambda_{i}\right.\right. \\
\left.\left.\left(1-\frac{(q+2) \delta_{i} \delta_{i}^{\prime}}{2 \sigma^{2} \Delta^{*} h_{i i}^{*}}\right) E\left(\chi_{q+4}^{-4}\left(\Delta^{*}\right)\right)\right]+v q \alpha_{i} \lambda_{i} \delta_{i} E\left(\chi_{q+2}^{-2}\left(\Delta^{*}\right)\right)-q v \alpha_{i} \delta_{i} E\left(\chi_{q+2}^{-2}\left(\Delta^{*}\right)\right)+\alpha_{i}^{2}\right\} & \\
f_{2}\left(\alpha, \Delta^{*}\right)= & \max _{1 \leq i \leq p}\left\{\sigma ^ { 2 } \left[\left(\frac{1}{\lambda_{i}}-v q(q-2) h_{i i}^{*} E\left(\chi_{q+2}^{-4}\left(\Delta^{*}\right)\right)\right)-2 q v \Delta^{*} h_{i i}^{*}\right.\right. \\
& \left.\left.\left(1-\frac{(q+2) \delta_{i}^{\prime} \delta_{i}}{2 \sigma^{2} \Delta^{*} h_{i i}^{*}}\right) E\left(\chi_{q+4}^{-4}\left(\Delta^{*}\right)\right)\right]-2 q v \alpha_{i} v \delta_{i} E\left(\chi_{q+2}^{-2}\left(\Delta^{*}\right)\right)+\alpha_{i}^{2}\right\} .
\end{aligned}
$$

Theorem 4.1.ii. A sufficient condition for SGRLE to have risk value less than or equal to GULE is that there exists a value of $d \in\left(0, d_{3}^{*}\right)$, where $d_{3}^{*}$ is given by

$$
d_{3}^{*}=\frac{f_{4}\left(\alpha_{i}, \Delta^{*}\right)}{f_{3}\left(\alpha_{i}, \Delta^{*}\right)}
$$

where,

$$
\begin{aligned}
& f_{3}\left(\alpha_{i}, \Delta^{*}\right)=\max _{1 \leq i \leq p}\left\{\sigma ^ { 2 } \left[-q v(q+2) h_{i i}^{*} E\left(\chi_{q+2}^{-4}\left(\Delta^{*}\right)\right)-2 q v \Delta^{*} h_{i i}^{*}\right.\right. \\
& \left.\left.\left(1-\frac{(q+2) \delta_{i}^{\prime} \delta_{i}}{2 \sigma^{2} \Delta^{*} h_{i i}^{*}}\right) E\left(\chi_{q+4}^{-4}\left(\Delta^{*}\right)\right)\right]-q v \alpha_{i} \delta_{i} E\left(\chi_{q+2}^{-2}\left(\Delta^{*}\right)\right)\right\} \\
& f_{4}\left(\alpha_{i}, \Delta^{*}\right)=\min _{1 \leq i \leq p}\left\{\sigma ^ { 2 } \left[q v(q+2) h_{i i}^{*} \lambda_{i} E\left(\chi_{q+2}^{-4}\left(\Delta^{*}\right)\right)+2 q v \Delta^{*} h_{i i}^{*} \lambda_{i}\right.\right. \\
& \left.\left.\left(1-\frac{(q+2) \delta_{i}^{\prime} \delta_{i}}{2 \sigma^{2} \Delta^{*} h_{i i}^{*}}\right) E\left(\chi_{q+4}^{-4}\left(\Delta^{*}\right)\right)\right]-q v \alpha_{i} \delta_{i} E\left(\chi_{q+2}^{-2}\left(\Delta^{*}\right)\right)\right\} .
\end{aligned}
$$


Theorem 4.1.iii. A sufficient condition for SGRLE to have smaller risk value than GRLE is that $d \in\left(0, d_{4}^{*}\right)$, where $d_{4}^{*}$ is given by:

$$
d_{4}^{*}=\frac{f_{6}\left(\alpha_{i}, \Delta^{*}\right)}{f_{5}\left(\alpha_{i}, \Delta^{*}\right)}
$$

where,

$$
\begin{aligned}
& f_{5}\left(\alpha_{i}, \Delta^{*}\right)=\min _{1 \leq i \leq p}\left\{\sigma ^ { 2 } \left[-v h_{i i}^{*} q(q-2) E\left(\chi_{q+2}^{-4}\left(\Delta^{*}\right)\right)-2 q v \Delta^{*} h_{i i}^{*}\right.\right. \\
& \left.\left.\left(1-\frac{(q+2) \delta_{i}^{\prime} \delta_{i}}{2 \sigma^{2} \Delta^{*} h_{i i}^{*}}\right) E\left(\chi_{q+4}^{-4}\left(\Delta^{*}\right)\right)+h_{i i}^{*}\right]-q v \alpha_{i} \delta_{i} \lambda_{i} E\left(\chi_{q+2}^{-2}\left(\Delta^{*}\right)\right)-\delta_{i}^{\prime} \delta_{i}\right\} \\
& f_{6}\left(\alpha_{i}, \Delta^{*}\right)=\max _{1 \leq i \leq p}\left\{\sigma ^ { 2 } \left[v h_{i i}^{*} \lambda_{i} q(q-2) E\left(\chi_{q+2}^{-4}\left(\Delta^{*}\right)\right)+2 q v \Delta^{*} h_{i i}^{*} \lambda_{i}\right.\right. \\
& \left.\left.\left(1-\frac{(q+2) \delta_{i}^{\prime} \delta_{i}}{2 \sigma^{2} \Delta^{*} h_{i i}^{*}}\right) E\left(\chi_{q+4}^{-4}\left(\Delta^{*}\right)\right)-h_{i i}^{*} \lambda_{i}\right]-q v \alpha_{i} \delta_{i} E\left(\chi_{q+2}^{-2}\left(\Delta^{*}\right)\right)+\delta_{i}^{\prime} \delta_{i} \lambda_{i}\right\} .
\end{aligned}
$$

Theorem 4.1.iv. A sufficient condition for SGRLE to have smaller risk value than PTGRLE is that $d \in\left(0, d_{5}^{*}\right)$, where $d_{5}^{*}$ is given by

$$
d_{5}^{*}=\frac{f_{8}\left(\alpha_{i}, \Delta^{*}\right)}{f_{7}\left(\alpha_{i}, \Delta^{*}\right)}
$$

where,

$$
\begin{aligned}
& f_{7}\left(\alpha_{i}, \Delta^{*}\right)=\min _{1 \leq i \leq p}\left[\sigma ^ { 2 } \left\{\frac{1}{\lambda_{i}}-v h_{i i}^{*} q(q-2) E\left(\chi_{q+2}^{-4}\left(\Delta^{*}\right)\right)-2 q v \Delta^{*} h_{i i}^{*}\right.\right. \\
& \left.\left(1-\frac{(q+2) \delta_{i}^{\prime} \delta_{i}}{2 \sigma^{2} \Delta^{*} h_{i i}^{*}}\right) E\left(\chi_{q+4}^{-4}\left(\Delta^{*}\right)\right)+h_{i i}^{*}\left(G_{q+2, n-p}\left(X^{\prime}, \Delta^{*}\right)\right)\right\}-2 q v \alpha_{i} \delta_{i} \\
& E\left(\chi_{q+2}^{-2}\left(\Delta^{*}\right)\right)-2 \alpha_{i} \delta_{i}\left(G_{q+2, n-p}\left(X^{\prime}, \Delta^{*}\right)\right)-\delta_{i}^{\prime} \delta_{i}\left[2 G_{q+2, n-p}\left(X^{\prime}, \Delta^{*}\right)\right. \\
& \left.\left.-G_{q+4, n-p}\left(X^{\prime}, \Delta^{*}\right)\right]\right] \\
& f_{8}\left(\alpha_{i}, \Delta^{*}\right)=\max _{1 \leq i \leq p} \sigma^{2}\left\{-1+v h_{i i}^{*} \lambda_{i} q(q-2) E\left(\chi_{q+2}^{-4}\left(\Delta^{*}\right)\right)+2 q v \Delta^{*} h_{i i}^{*} \lambda_{i}\right. \\
& \left(1-\frac{(q+2) \delta_{i}^{\prime} \delta_{i}}{2 \sigma^{2} \Delta^{*} h_{i i}^{*}} E\left(\chi_{q+4}^{-4}\left(\Delta^{*}\right)\right)-h_{i i}^{*} \lambda_{i}\left(G_{q+2, n-p}\left(X^{\prime}, \Delta^{*}\right)\right)\right\} \\
& -2 q v \alpha_{i} \delta_{i} E\left(\chi_{q+2}^{-2}\left(\Delta^{*}\right)\right)+\lambda_{i} \delta_{i}^{\prime} \delta_{i}\left[2 G_{q+2, n-p}\left(X^{\prime}, \Delta^{*}\right)-G_{q+4, n-p}\left(X^{\prime}, \Delta^{*}\right)\right] .
\end{aligned}
$$

Theorem 4.1.v. There always exist a positive $d \in\left(0, d_{6}^{*}\right)$ and $\left(0, d_{7}^{*}\right)$ such that PRSGRLE has smaller risk value than PRSGRE under $H_{0}: H \beta=h$ and $H_{A}: H \beta \neq h$, respectively, where

$$
\begin{gathered}
d_{6}^{*}=\frac{f_{10}\left(\alpha_{i}, \Delta^{*}\right)}{f_{9}\left(\alpha_{i}, \Delta^{*}\right)} \\
d_{7}^{*}=\frac{f_{12}\left(\alpha_{i}, \Delta^{*}\right)}{f_{11}\left(\alpha_{i}, \Delta^{*}\right)}
\end{gathered}
$$

where,

$$
\begin{aligned}
& f_{9}\left(\alpha_{i}, \Delta^{*}\right)=\max _{1 \leq i \leq p}\left\{\sigma ^ { 2 } \left[\frac{1}{\lambda_{i}}-v h_{i i}^{*}-h_{i i}^{*} E\left[\left(1-\frac{q v}{q+2} F_{q+2, n-p}^{-1}\left(\Delta^{*}\right)\right)^{2}\right.\right.\right. \\
& \left.\left.I\left(F_{q+2, n-p}\left(\Delta^{*}\right) \leq \frac{q v}{q+2}\right)\right]\right]+\alpha_{i}^{2} \\
& \left.f_{10}\left(\alpha_{i}, \Delta^{*}\right)\right\}=\min _{1 \leq i \leq p}\left\{\sigma ^ { 2 } \left[v h_{i i}^{*} \lambda_{i}+h_{i i}^{*} \lambda_{i} E\left[\left(1-\frac{q v}{q+2} F_{q+2, n-p}^{-1}\left(\Delta^{*}\right)\right)^{2}\right.\right.\right. \\
& \left.\left.\left.I\left(F_{q+2, n-p}\left(\Delta^{*}\right) \leq \frac{q v}{q+2}\right)\right]-1\right]+\alpha_{i}^{2}\right\}
\end{aligned}
$$




$$
\begin{aligned}
& f_{11}\left(\alpha_{i}, \Delta^{*}\right)=\max _{1 \leq i \leq p}\left\{\sigma ^ { 2 } \left[\frac{1}{\lambda_{i}}-q v(q+2) h_{i i}^{*} E\left(\chi_{q+2}^{-4}\left(\Delta^{*}\right)\right)-2 q v h_{i i}^{*} \Delta^{*}\right.\right. \\
& \left(1-\frac{(q+2) \delta_{i}^{\prime} \delta_{i}}{2 \sigma^{2} \Delta^{*} h_{i i}^{*}}\right) E\left(\chi_{q+4}^{-4}\left(\Delta^{*}\right)\right)-h_{i i}^{*} E\left(1-\frac{q v}{q+2} F_{q+2, n-p}^{-1}\left(\Delta^{*}\right)\right)^{2} \\
& \left.I\left(F_{q+2, n-p}\left(\Delta^{*}\right) \leq \frac{q v}{q+2}\right)\right]-q v \delta_{i} \alpha_{i} E\left(\chi_{q+2}^{-2}\left(\Delta^{*}\right)\right)+\alpha_{i} \\
& +\delta_{i}^{\prime} \delta_{i} E\left[\left(1-\frac{q v}{q+4} F_{q+4, n-p}^{-1}\left(\Delta^{*}\right)\right)^{2} I\left(F_{q+4, n-p}\left(\Delta^{*}\right) \leq \frac{q v}{q+4}\right)\right] \\
& -2 \delta_{i}^{\prime} \delta_{i} E\left[\left(\frac{q v}{q+2} F_{q+2, n-p}^{-1}\left(\Delta^{*}\right)-1\right) I\left(F_{q+2, n-p}\left(\Delta^{*}\right) \leq \frac{q v}{q+2}\right)\right] \\
& \left.+\delta_{i}^{\prime} \alpha_{i} E\left[\left(\frac{q v}{q+2} F_{q+2, n-p}^{-1}\left(\Delta^{*}\right)-1\right) I\left(F_{q+2, n-p}\left(\Delta^{*}\right) \leq \frac{q v}{q+2}\right)\right]\right\} \\
& f_{12}\left(\alpha_{i}, \Delta^{*}\right)=\min _{1 \leq i \leq p}\left\{\sigma ^ { 2 } \left[-1+q v(q+2) h_{i i}^{*} \lambda_{i} E\left(\chi_{q+2}^{-4}\left(\Delta^{*}\right)\right)\right.\right. \\
& +2 q v h_{i i}^{*} \lambda_{i} \Delta^{*}\left(1-\frac{(q+2) \delta_{i} \delta_{i}^{\prime}}{2 \sigma^{2} \Delta^{*} h_{i i}^{*}}\right) E\left(\chi_{q+4}^{-4}\left(\Delta^{*}\right)\right)+h *_{i i} \lambda_{i} \\
& \left.E\left(1-\frac{q v}{q+2} F_{q+2, n-p}^{-1}\left(\Delta^{*}\right)\right)^{2} I\left(F_{q+2, n-p}\left(\Delta^{*}\right) \leq \frac{q v}{q+2}\right)\right]-q v \delta_{i} \alpha_{i} \lambda_{i} \\
& E\left(\chi_{q+2}^{-2}\left(\Delta^{*}\right)\right)+q v \delta_{i} \alpha_{i} E\left(\chi_{q+2}^{-2}\left(\Delta^{*}\right)\right)+\alpha_{i}^{2}-\delta_{i}^{\prime} \delta_{i} \lambda_{i} \\
& E\left[\left(1-\frac{q v}{q+4} F_{q+4, n-p}^{-1}\left(\Delta^{*}\right)\right)^{2} I\left(F_{q+4, n-p}\left(\Delta^{*}\right) \leq \frac{q v}{q+4}\right)\right]+2 \delta_{i}^{\prime} \delta_{i} \lambda_{i} \\
& E\left[\left(\frac{q v}{q+2} F_{q+2, n-p}^{-1}\left(\Delta^{*}\right)-1\right) I\left(F_{q+2, n-p}\left(\Delta^{*}\right) \leq \frac{q v}{q+2}\right)\right] \\
& +\delta_{i}^{\prime} \alpha_{i} E\left[\left(\frac{q v}{q+2} F_{q+2, n-p}^{-1}\left(\Delta^{*}\right)-1\right) I\left(F_{q+2, n-p}\left(\Delta^{*}\right) \leq \frac{q v}{q+2}\right)\right] \\
& \left.-\delta_{i}^{\prime} \alpha_{i} E\left[\left(\frac{q v}{q+2} F_{q+2, n-p}^{-1}\left(\Delta^{*}\right)-1\right) I\left(F_{q+2, n-p}\left(\Delta^{*}\right) \leq \frac{q d}{q+2}\right)\right]\right\} .
\end{aligned}
$$

Theorem 4.1.vi. PRSGRLE has smaller risk value than SGRLE for all positive $d$.

Theorem 4.1.vii. A sufficient condition for PRSGRLE to have smaller risk value than or equal to GULE is that $d \in\left(0, d_{3}^{*}\right)$, where $d_{3}^{*}$ is given by (14).

Theorem 4.1.viii. A sufficient condition for PRSGRLE to have smaller risk value than GRLE is that $d \in\left(0, d_{8}^{*}\right)$, where $d_{8}^{*}$ is given by

$$
d_{8}^{*}=\frac{f_{14}\left(\alpha_{i}, \Delta^{*}\right)}{f_{13}\left(\alpha_{i}, \Delta^{*}\right)}
$$

where,

$$
\begin{aligned}
& f_{13}\left(\alpha_{i}, \Delta^{*}\right)=\min _{1 \leq i \leq p}\left\{\sigma ^ { 2 } \left[-v h_{i i}^{*} q(q-2) E\left(\chi_{q+2}^{-4}\left(\Delta^{*}\right)\right)-2 v \Delta^{*} h_{i i}^{*} q\right.\right. \\
& \left(1-\frac{(q+2) \delta_{i}^{\prime} \delta_{i}}{2 \sigma^{2} \Delta^{*} h_{i i}^{*}}\right) E\left[\left(\chi_{q+4}^{-4}\left(\Delta^{*}\right)\right)\right]-h_{i i}^{*} E\left[\left(1-\frac{q v}{q+2} F_{q+2, n-p}^{-1}\left(\Delta^{*}\right)\right)^{2}\right. \\
& \left.\left.I\left(F_{q+2, n-p}\left(\Delta^{*}\right) \leq \frac{q v}{q+2}\right)\right]+h_{i i}^{*}\right]-q v \alpha_{i} \delta_{i} E\left(\chi_{q+2}^{-2}\left(\Delta^{*}\right)\right)+\delta_{i}^{\prime} \delta_{i} \\
& E\left[\left(1-\frac{q v}{q+4} F_{q+4, n-p}^{-1}\left(\Delta^{*}\right)\right)^{2} I\left(F_{q+4, n-p}\left(\Delta^{*}\right) \leq \frac{q v}{q+4}\right)\right]
\end{aligned}
$$




$$
\begin{array}{rl}
-2 \delta_{i}^{\prime} \delta_{i} & E\left[\left(\frac{q v}{q+2} F_{q+2, n-p}^{-1}\left(\Delta^{*}\right)-1\right) I\left(F_{q+2, n-p}\left(\Delta^{*}\right) \leq \frac{q v}{q+2}\right)\right]+\delta_{i}^{\prime} \alpha_{i} \\
& \left.E\left[\left(\frac{q v}{q+2} F_{q+2, n-p}^{-1}\left(\Delta^{*}\right)-1\right) I\left(F_{q+2, n-p}\left(\Delta^{*}\right) \leq \frac{q v}{q+2}\right)\right]-\delta_{i}^{\prime} \delta_{i}\right\} \\
& f_{14}\left(\alpha_{i}, \Delta^{*}\right)=\max _{1 \leq i \leq p}\left\{\sigma ^ { 2 } \left[h_{i i}^{*} \lambda_{i} q(q-2) E\left(\chi_{q+2}^{-4}\left(\Delta^{*}\right)\right)+2 v \Delta^{*} q h_{i i}^{*} \lambda_{i}\right.\right. \\
& \left(1-\frac{(q+2) \delta_{i}^{\prime} \delta_{i}}{2 \sigma^{2} \Delta^{*} h_{i i}^{*}}\right) E\left[\left(\chi_{q+4}^{-4}\left(\Delta^{*}\right)\right)\right]+h_{i i}^{*} \lambda_{i} E\left[\left(1-\frac{q v}{q+2} F_{q+2, n-p}^{-1}\left(\Delta^{*}\right)\right)^{2}\right. \\
& \left.\left.I\left(F_{q+2, n-p}\left(\Delta^{*}\right) \leq \frac{q v}{q+2}\right)\right]-h_{i i}^{*} \lambda_{i}\right]-\delta_{i}^{\prime} \delta_{i} \lambda_{i} E\left[\left(1-\frac{q v}{q+4} F_{q+4, n-p}^{-1}\left(\Delta^{*}\right)\right)^{2}\right. \\
& \left.I\left(F_{q+4, n-p}\left(\Delta^{*}\right) \leq \frac{q v}{q+4}\right)\right]+2 \delta_{i}^{\prime} \delta_{i} \lambda_{i} E\left[\left(\frac{q v}{q+2} F_{q+2, n-p}^{-1}\left(\Delta^{*}\right)-1\right)\right. \\
& \left.I\left(F_{q+2, n-p}\left(\Delta^{*}\right) \leq \frac{q v}{q+2}\right)\right]+\delta_{i}^{\prime} \alpha_{i} E\left[\left(\frac{q v}{q+2} F_{q+2, n-p}^{-1}\left(\Delta^{*}\right)-1\right)\right. \\
& \left.\left.I\left(F_{q+2, n-p}\left(\Delta^{*}\right) \leq \frac{q v}{q+2}\right)\right]+\delta_{i}^{\prime} \delta_{i} \lambda_{i}-q v \alpha_{i} \delta E\left[\left(\chi_{q+2}^{-2}\left(\Delta^{*}\right)\right)\right]\right\}
\end{array}
$$

Theorem 4.1.ix. A sufficient condition for PRSGRLE to have smaller risk value than PTGRLE is that is that $d \in\left(0, d_{9}^{*}\right)$, where $d_{9}^{*}$ is given by

$$
d_{9}^{*}=\frac{f_{15}\left(\alpha, \Delta^{*}\right)}{f_{16}\left(\alpha, \Delta^{*}\right)}
$$

where,

$$
\begin{aligned}
& f_{16}\left(\alpha, \Delta^{*}\right)=\min _{1 \leq i \leq p}\left\{\sigma ^ { 2 } \left\{\frac{1}{\lambda_{i}}-v h_{i i}^{*} q(q-2) E\left(\chi_{q+2}^{-4}\left(\Delta^{*}\right)\right)-2 q v \Delta^{*} h_{i i}^{*}\right.\right. \\
& \left(1-\frac{(q+2) \delta_{i}^{\prime} \delta_{i}}{2 \sigma^{2} \Delta^{*} h_{i i}^{*}}\right) E\left(\chi_{q+4}^{-4}\left(\Delta^{*}\right)\right)-h_{i i}^{*} E\left[\left(1-\frac{q v}{q+2} F_{q+2, n-p}^{-1}\left(\Delta^{*}\right)\right)^{2}\right. \\
& \left.\left.I\left(F_{q+2, n-p}\left(\Delta^{*}\right) \leq \frac{q v}{q+2}\right)\right]+h_{i i}^{*} v\left(G_{q+2 . n-p}\left(X^{\prime}, \Delta^{*}\right)\right)\right\}-q v \alpha_{i} \delta_{i} \\
& E\left[\left(\chi_{q+2}^{-2}\left(\Delta^{*}\right)\right)\right]+\delta_{i}^{\prime} \delta_{i} E\left[\left(1-\frac{q v}{q+4} F_{q+4, n-p}^{-1}\left(\Delta^{*}\right)\right)^{2} I\left(F_{q+4, n-p}\left(\Delta^{*}\right) \leq \frac{q v}{q+4}\right)\right] \\
& -2 \delta_{i}^{\prime} \delta_{i} E\left(\frac{q v}{q+2} F_{q+2, n-p}^{-1}\left(\Delta^{*}\right)-1\right) I\left(F_{q+2, n-p}\left(\Delta^{*}\right) \leq \frac{q v}{q+2}\right)+\delta_{i}^{\prime} \alpha_{i} \\
& E\left(\frac{q v}{q+2} F_{q+2, n-p}^{-1}\left(\Delta^{*}\right)-1\right) I\left(F_{q+2, n-p}\left(\Delta^{*}\right) \leq \frac{q v}{q+2}\right)-2 \alpha_{i} \delta_{i} \\
& \left.\left(G_{q+2, n-p}\left(X^{\prime}, \Delta^{*}\right)\right)-\delta_{i}^{\prime} \delta_{i}\left(2 G_{q+2 . n-p}\left(X^{\prime}, \Delta^{*}\right)-G_{q+2 . n-p}\left(X^{\prime}, \Delta^{*}\right)\right)\right\}
\end{aligned}
$$




$$
\begin{aligned}
& f_{15}\left(\alpha, \Delta^{*}\right)=\max _{1 \leq i \leq p}\left\{\sigma ^ { 2 } \left\{v h_{i i}^{*} \lambda_{i} q(q-2) E\left(\chi_{q+2}^{-4}\left(\Delta^{*}\right)\right)+2 q v \Delta^{*} h_{i i}^{*} \lambda_{i}\right.\right. \\
& \left(1-\frac{(q+2) \delta_{i}^{\prime} \delta_{i}}{2 \sigma^{2} \Delta^{*} h_{i i}^{*}}\right) E\left(\chi_{q+4}^{-4}\left(\Delta^{*}\right)\right)+h_{i i}^{*} \lambda_{i} E\left[\left(1-\frac{q v}{q+2} F_{q+2, n-p}^{-1}\left(\Delta^{*}\right)\right)^{2}\right. \\
& \left.\left.I\left(F_{q+2, n-p}\left(\Delta^{*}\right) \leq \frac{q v}{q+2}\right)\right]-\lambda_{i} h_{i i}^{*}\left(G_{q+2 . n-p}\left(X^{\prime}, \Delta^{*}\right)\right)-1\right\}-\delta_{i}^{\prime} \delta_{i} \lambda_{i} \\
& E\left[\left(1-\frac{q v}{q+4} F_{q+4, n-p}^{-1}\left(\Delta^{*}\right)\right)^{2} I\left(F_{q+4, n-p}\left(\Delta^{*}\right) \leq \frac{q v}{q+4}\right)\right]+2 \delta_{i}^{\prime} \delta_{i} \lambda_{i} \\
& E\left(\frac{q v}{q+2} F_{q+2, n-p}^{-1}\left(\Delta^{*}\right)-1\right) I\left(F_{q+2, n-p}\left(\Delta^{*}\right) \leq \frac{q v}{q+2}\right)+\delta_{i}^{\prime} \alpha_{i} \\
& E\left(\frac{q v}{q+2} F_{q+2, n-p}^{-1}\left(\Delta^{*}\right)-1\right) I\left(F_{q+2, n-p}\left(\Delta^{*}\right) \leq \frac{q v}{q+2}\right)+\lambda_{i} \delta_{i}^{\prime} \delta_{i} \\
& \left.\left(2 G_{q+2 . n-p}\left(X^{\prime}, \Delta^{*}\right)-G_{q+2 . n-p}\left(X^{\prime}, \Delta^{*}\right)\right)-q v \alpha_{i} \delta_{i} E\left[\left(\chi_{q+2}^{-2}\left(\Delta^{*}\right)\right)\right]\right\} .
\end{aligned}
$$

Proof

We only prove the first and last theorems. The proofs of the others are similar and just require a straight forward calculation.

Proof of Theorem 4.1.i: Using Eqs.(11)-(13) under $H_{0}: H \beta \neq h$, the risk expression is given by

$$
R\left(\hat{\beta}_{G R}^{S}(d) ; \beta\right)=\sum_{i=1}^{p} \frac{1}{\left(\lambda_{i}+1\right)^{2}}\left[(1-d)^{2} \alpha_{i}^{2}+\sigma^{2}\left(\frac{\left(\lambda_{i}+d\right)^{2}}{\lambda_{i}}-v h_{i i}^{*}\left(\lambda_{i}+d\right)^{2}\right)\right] .
$$

Differentiating with respect to $d$ we find

$$
\frac{\partial R\left(\hat{\beta}_{G R}(d) ; \beta\right)}{\partial d}=\sum_{i=1}^{p} \frac{\left(-2(1-d) \alpha_{i}^{2}+2 \sigma^{2}\left(\lambda_{i}+d\right)\left(\frac{1}{\lambda_{i}}-v h_{i i}^{*}\right)\right.}{\left(\lambda_{i}+1\right)^{2}} .
$$

Thus, a sufficient condition for (16) to be negative is that $0<d<d_{1}^{*}$ where

$$
d_{1}^{*}=\frac{\min \left(v h_{i i}^{*} \lambda_{i}-1+\alpha_{i}^{2}\right)}{\max \left(\frac{\alpha_{i}^{2}}{\sigma^{2}}+\frac{1}{\lambda_{i}}-v h_{i i}^{*}\right)} .
$$

Now, under $H_{A}: H \beta \neq h$, the risk expression is given by

$$
\begin{aligned}
& R\left(\hat{\beta}_{G R}^{S}(d) ; \beta\right)=\sum_{i=1}^{p} \frac{1}{\left(\lambda_{i}+I\right)^{2}}\left\{\sigma ^ { 2 } \left[\frac{\left(\lambda_{i}+d\right)^{2}}{\lambda_{i}}-v h_{i i}^{*} q\left(\lambda_{i}+d\right)^{2}\left\{(q-2) E\left(\chi_{q+2}^{-4}\left(\Delta^{*}\right)\right)\right.\right.\right. \\
& \left.+2 \Delta^{*}\left[1-\frac{(q+2) \delta_{i}^{\prime} \delta_{i}\left(\lambda_{i}+d\right)^{2}}{2 \sigma^{2} \Delta^{*} h_{i i}^{*}\left(\lambda_{i}+d\right)^{2}}\right] E\left(\chi_{q+4}^{-4}\left(\Delta^{*}\right)\right)\right\}+2 q v(1-d) \alpha_{i}\left(\lambda_{i}+d\right) \delta_{i} E\left(\chi_{q+2}^{-2}\left(\Delta^{*}\right)\right) \\
& \left.+(1-d)^{2} \alpha_{i}^{2}\right\}
\end{aligned}
$$

in which $\delta_{i}$ is the $i$ th element of $\delta$, Differentiating with respect to $d$, gives

$$
\begin{gathered}
\frac{\partial R\left(\hat{\beta}_{G R}^{S}(d) ; \beta\right)}{\partial d}=\sum_{i=1}^{p} \frac{1}{\left(\lambda_{i}+1\right)^{2}} \sigma^{2}\left[\frac{\left(\lambda_{i}+d\right)}{\lambda_{i}}-q v h_{i i}^{*}\left(\lambda_{i}+d\right)\left\{(q-2) E\left(\chi_{q+2}^{-4}\left(\Delta^{*}\right)\right)+\right.\right. \\
\left.\left.\left(1-\frac{(q+2) \delta_{i}^{\prime} \delta_{i}}{2 \sigma^{2} \Delta^{*} h_{i i}^{*}}\right) E\left(\chi_{q+4}^{-4}\left(\Delta^{*}\right)\right)\left(2 \Delta^{*}\right)\right\}\right]+q v \alpha_{i} \delta_{i} E\left(\chi_{q+2}^{-2}\left(\Delta^{*}\right)\right)-(1-d) \alpha_{i}^{2} \\
f_{1}\left(\alpha, \Delta^{*}\right)=\min _{1 \leq i \leq p}\left[\left(\alpha_{i}^{2}-\sigma^{2}+q v(q-2) \sigma^{2} h_{i i}^{*} \lambda_{i} E\left(\chi_{q+2}^{-4}\left(\Delta^{*}\right)\right)\right)+2 q v \sigma^{2} \Delta^{*} h_{i i}^{*} \lambda_{i}\right. \\
\left.\left(1-\frac{(q+2) \delta_{i}^{\prime} \delta_{i}}{2 \sigma^{2} \Delta^{*} h_{i i}^{*}}\right) E\left(\chi_{q+4}^{-4}\left(\Delta^{*}\right)\right)+q v \alpha_{i} \lambda_{i} \delta_{i} E\left(\chi_{q+2}^{-2}\left(\Delta^{*}\right)\right)-q v \alpha_{i} \delta_{i} E\left(\chi_{q+2}^{-2}\left(\Delta^{*}\right)\right)\right]
\end{gathered}
$$




$$
\begin{aligned}
& \begin{aligned}
f_{2}\left(\alpha, \Delta^{*}\right)= & \max _{1 \leq i \leq p}\left(\left(\frac{\sigma^{2}}{\lambda_{i}}-v q(q-2) \sigma^{2} h_{i i}^{*} E\left(\chi_{q+2}^{-4}\left(\Delta^{*}\right)\right)\right)-2 q v \sigma^{2} \Delta^{*} h_{i i}^{*}\right. \\
& \left.\left(1-\frac{(q+2) \delta_{i}^{\prime} \delta_{i}}{2 \sigma^{2} \Delta^{*} h_{i i}^{*}}\right) E\left(\chi_{q+4}^{-4}\left(\Delta^{*}\right)\right)-q v \alpha_{i} \delta_{i} E\left(\chi_{q+2}^{-2}\left(\Delta^{*}\right)\right)+\alpha_{i}^{2}\right)
\end{aligned} \\
& d_{2}^{*}=\frac{f_{1}\left(\alpha, \Delta^{*}\right)}{f_{2}\left(\alpha, \Delta^{*}\right)}
\end{aligned}
$$

Hence, a sufficient for (17) to be negative is that $0<d<d_{2}^{*}$. Thus, $\hat{\beta}_{G R}^{S}(d)$ has risk value less than the risk of $\hat{\beta}^{S}$ for $0<d<d_{2}^{*}$.

Proof of Theorem 4.1.ix: By making use of risk difference we obtain

$$
\begin{aligned}
& R\left(\hat{\beta}_{G R}^{S+}(d) ; \beta\right)-R\left(\hat{\beta}_{G R}^{P T}(d) ; \beta\right)=\sum_{i=1}^{p} \frac{1}{\left(\lambda_{i}+1\right)^{2}}\left\{\sigma ^ { 2 } \left[\frac{\left(\lambda_{i}+d\right)^{2}}{\lambda_{i}}-v h_{i i}^{*}\left(\lambda_{i}+d\right)^{2}\right.\right. \\
& \left.q(q-2) E\left(\chi_{q+2}^{-4}\left(\Delta^{*}\right)\right)-2 q v \Delta^{*} h_{i i}^{*}\left(\lambda_{i}+d\right)^{2}\left(1-\frac{(q+2) \delta_{i}^{\prime} \delta_{i}}{2 \sigma^{2} \Delta * h_{i i}^{*}}\right) E\left(\chi_{q+4}^{-4}\left(\Delta^{*}\right)\right)\right] \\
& +2 q v(1-d) \alpha_{i}\left(\lambda_{i}+d\right) \delta_{i} E\left(\chi_{q+2}^{-2}\left(\Delta^{*}\right)\right)+(1-d)^{2} \alpha_{i}^{2}-\sigma^{2} h_{i i}^{*}\left(\lambda_{i}+d\right)^{2} \\
& E\left[\left(1-\frac{q v}{q+2} F_{q+2, n-p}^{-1}\left(\Delta^{*}\right)\right)^{2} I\left(F_{q+2, n-p}\left(\Delta^{*}\right) \leq \frac{q v}{q+2}\right)\right]+\delta_{i}^{\prime} \delta_{i}\left(\lambda_{i}+d\right)^{2} \\
& E\left[\left(1-\frac{q v}{q+4} F_{q+4, n-p}^{-1}\left(\Delta^{*}\right)\right)^{2} I\left(F_{q+4, n-p}\left(\Delta^{*}\right) \leq \frac{q v}{q+4}\right)\right]-2 \delta_{i}^{\prime} \delta_{i}\left(\lambda_{i}+d\right)^{2} \\
& E\left[\left(\frac{q v}{q+2} F_{q+2, n-p}^{-1}\left(\Delta^{*}\right)-1\right) I\left(F_{q+2, n-p}\left(\Delta^{*}\right) \leq \frac{q v}{q+2}\right)\right]-2(1-d) \delta_{i}^{\prime} \alpha_{i}\left(\lambda_{i}+d\right) \\
& E\left[\left(\frac{q v}{q+2} F_{q+2, n-p}^{-1}\left(\Delta^{*}\right)-1\right) I\left(F_{q+2, n-p}\left(\Delta^{*}\right) \leq \frac{q v}{q+2}\right)\right]-2 \frac{\left(\lambda_{i}+d\right)^{2}}{\lambda_{i}} \\
& v \alpha_{i} \delta_{i}\left(G_{q+2 . n-p}\left(X^{\prime}, \Delta^{*}\right)\right)+\sigma^{2} h_{i i}^{*}\left(\lambda_{i}+d\right)^{2}\left(G_{q+2 . n-p}\left(X^{\prime}, \Delta^{*}\right)\right) \\
& \left.-\left(\lambda_{i}+d\right)^{2} \delta_{i}^{\prime} \delta_{i}\left(2 G_{q+2 . n-p}\left(X^{\prime}, \Delta^{*}\right)-G_{q+2 . n-p}\left(X^{\prime}, \Delta^{*}\right)\right)+(1-d)^{2} \alpha_{i}^{2}\right\} .
\end{aligned}
$$

This difference is non positive $(\leq 0)$, whenever

$$
d_{9}^{*}=\frac{f_{15}\left(\alpha, \Delta^{*}\right)}{f_{16}\left(\alpha, \Delta^{*}\right)}
$$

\section{Simulation Study}

In this section, we examine the risk and bias performances of the proposed estimators numerically. To achieve different degrees of collinearity, following McDonald [14] and Gibbons [7] the explanatory variables were generated using the following device

$$
x_{i j}=\left(1-\gamma^{2}\right)^{\frac{1}{2}} z_{i j}+\gamma z_{i j}
$$

where $z_{i j}$ are independent standard normal pseudo-random numbers, and $\gamma$ is specified so that the correlation between any two explanatory variables is given by $\gamma^{2}$. 

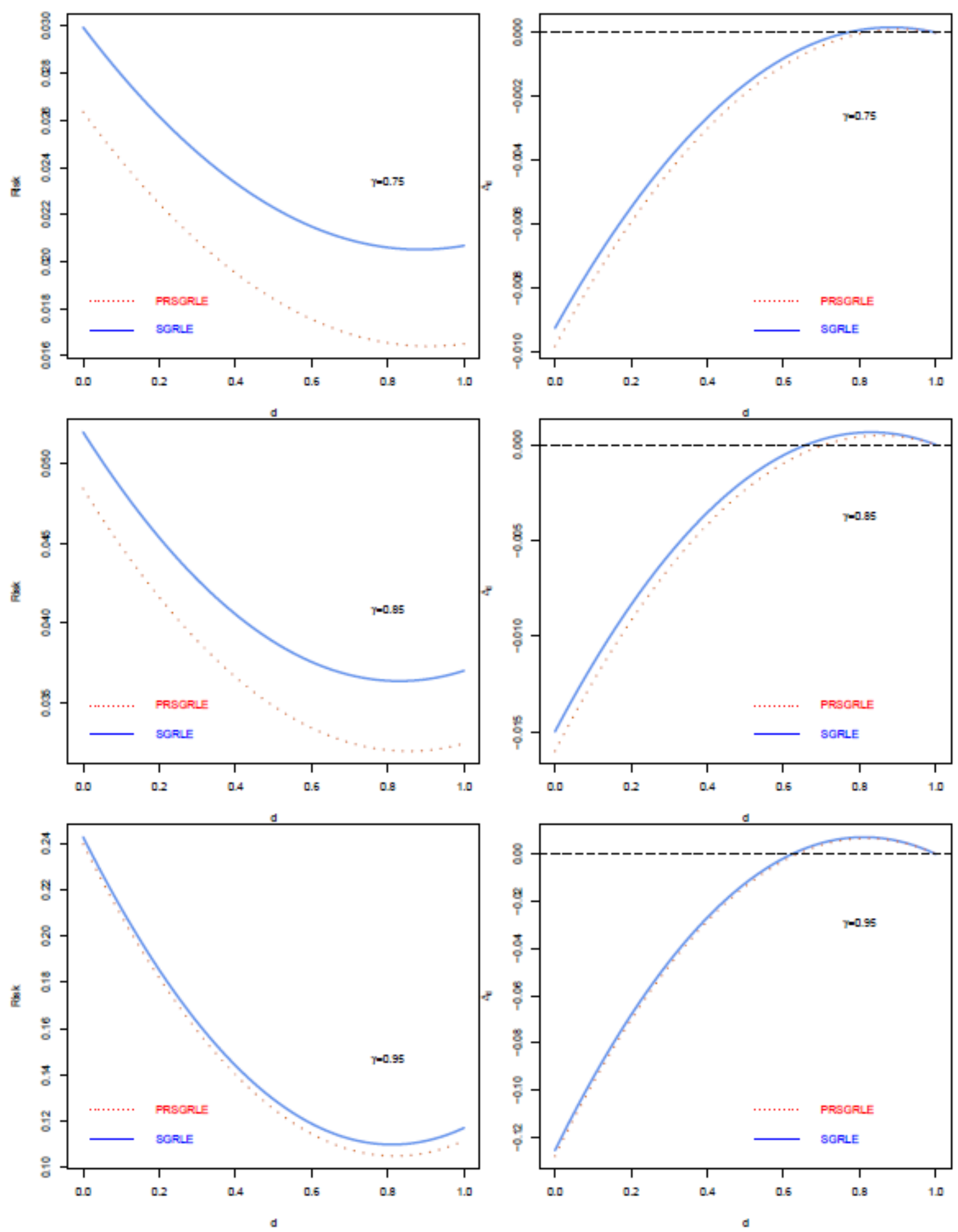

Figure 1. The diagrams of $R($.$) and \Delta_{d}$ versus $d$ for different values of $\gamma$. 

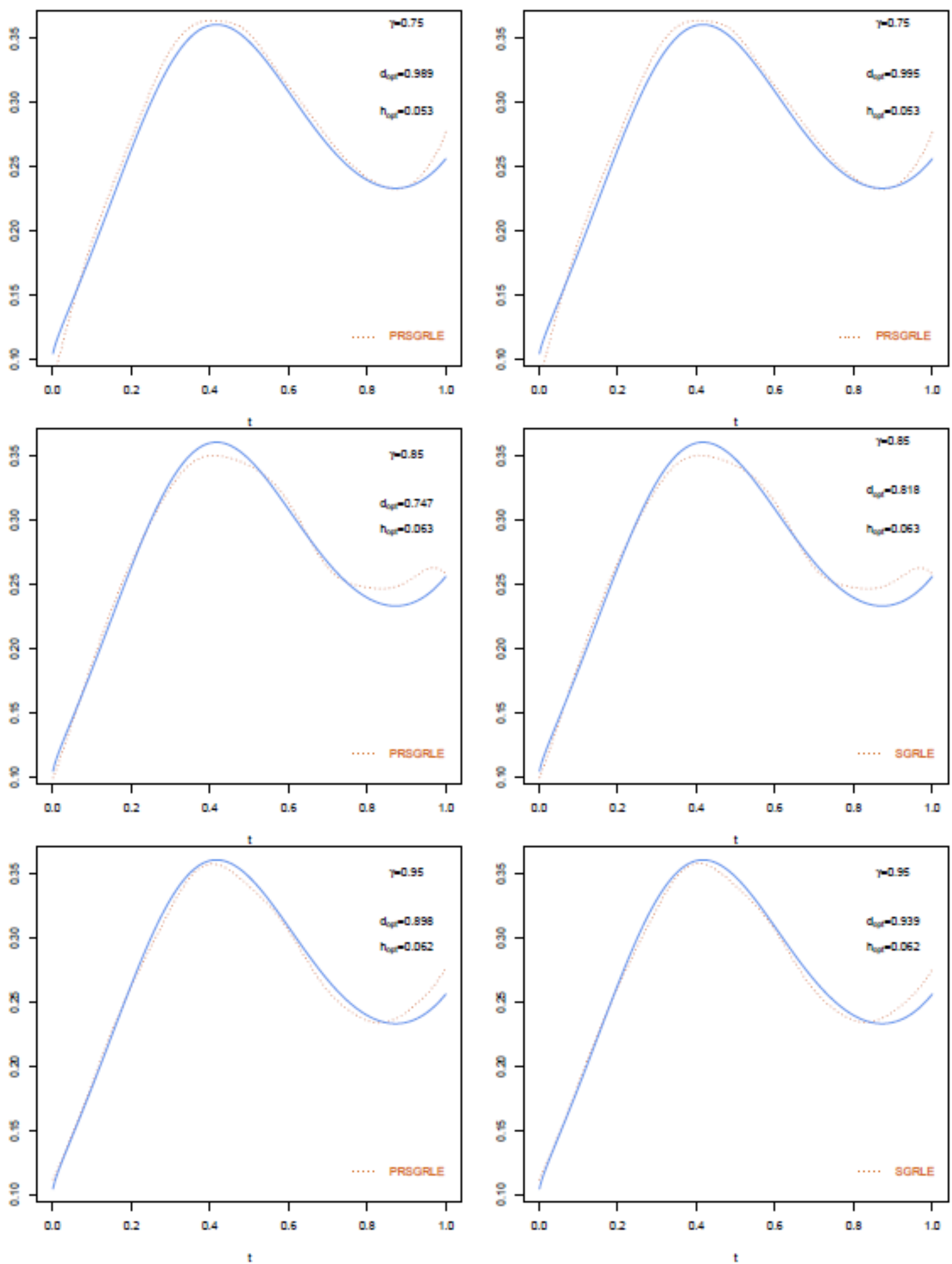

Figure 2. Estimation of the function under study by kernel approach for $n=500$. Red dashed lines are the estimates and blue solid lines are the true functions

Stat., Optim. Inf. Comput. Vol. 6, September 2018 
Table 1. Evaluation of $S G R L E$ at different $d$ values in model (20) with $\gamma=0.75$.

\begin{tabular}{cccccccc}
\hline$d$ & 0 & 0.2 & 0.4 & 0.6 & 0.8 & $d_{\text {opt }}=0.8888$ & 1 \\
\hline$\hat{\beta}_{1}$ & 0.9483368 & 0.9487535 & 0.9491702 & 0.9495869 & 0.9500037 & 0.9666719 & 0.968963 \\
$\hat{\beta}_{2}$ & 0.0652317 & 0.0650004 & 0.0647690 & 0.0645376 & 0.0643062 & 0.0550511 & 0.053778 \\
$\hat{\beta}_{3}$ & -0.9007455 & -0.9022973 & -0.9038491 & -0.9054008 & -0.9069526 & -0.9690238 & -0.977558 \\
$\hat{\beta}_{4}$ & 0.8731342 & 0.8744688 & 0.8758034 & 0.8771380 & 0.8784726 & 0.9318563 & 0.9391966 \\
$Q B\left[\hat{\beta}_{G R}^{S}(d)\right]$ & 0.0328706 & 0.0321551 & 0.0314484 & 0.03075062 & 0.3006162 & 0.0097444 & 0.0080560 \\
$R\left[\hat{\beta}_{G R}^{S}(d), \beta\right]$ & 0.0299489 & 0.0295226 & 0.0291062 & 0.0286996 & 0.0283029 & 0.0205151 & 0.0206773 \\
$\Delta$ & -0.0092715 & -0.0088452 & -0.0084288 & -0.0080222 & -0.0076255 & 0.0001622 & 0.0000000 \\
$m s e(\hat{f})$ & 0.0217902 & 0.0217886 & 0.0217840 & 0.0217808 & 0.0217724 & 0.0217513 & 0.0217561 \\
\hline \hline
\end{tabular}

Table 2. Evaluation of PRSGRLE at different $d$ values in model (20) with $\gamma=0.75$.

\begin{tabular}{cccccccc}
\hline$d$ & 0 & 0.2 & 0.4 & 0.6 & 0.8 & $d_{\text {opt }}=0.9090$ & 1 \\
\hline$\hat{\beta}_{1}$ & 0.9634681 & 0.9638791 & 0.9642901 & 0.9647011 & 0.9651121 & 0.9819628 & 0.9838123 \\
$\hat{\beta}_{2}$ & 0.0275543 & 0.0272764 & 0.0269986 & 0.0267207 & 0.0264428 & 0.0150505 & 0.0138001 \\
$\hat{\beta}_{3}$ & -0.9272026 & -0.9288097 & -0.9304168 & -0.9320239 & -0.9336310 & -0.9995216 & -1.0067535 \\
$\hat{\beta}_{4}$ & 0.9223636 & 0.9237903 & 0.9252170 & 0.9266437 & 0.9280704 & 0.9865646 & 0.9929847 \\
$Q B\left[\hat{\beta}_{G R}^{S}(d)\right]$ & 0.0134206 & 0.0129246 & 0.0124384 & 0.0119619 & 0.0114950 & 0.0007325 & 0.0005473 \\
$R\left[\hat{\beta}_{G R}^{S}(d), \beta\right]$ & 0.0263479 & 0.0259105 & 0.0254831 & 0.0250654 & 0.0246576 & 0.0164047 & 0.0165057 \\
$\Delta$ & -0.0098421 & -0.0094047 & -0.0089773 & -0.0085596 & -0.0081518 & 0.0001010 & 0.0000000 \\
$m s e(f)$ & 0.09815568 & 0.0872697 & 0.0799846 & 0.0796721 & 0.0795231 & 0.0706455 & 0.0815417 \\
\hline \hline
\end{tabular}

Table 3. Evaluation of SGRLE at different $d$ values in model (20) with $\gamma=0.85$.

\begin{tabular}{cccccccc}
\hline$d$ & 0 & 0.2 & 0.4 & 0.6 & 0.8 & $d_{\text {opt }}=0.8282$ & 1 \\
\hline$\hat{\beta}_{1}$ & 1.063341 & 1.0643487 & 1.0653734 & 1.0663981 & 1.0674227 & 1.1053353 & 1.1140449 \\
$\hat{\beta}_{2}$ & -0.1003593 & -0.1006017 & -0.1008441 & -0.1010865 & -0.1013289 & -0.1102974 & -0.1123577 \\
$\hat{\beta}_{3}$ & -0.8182077 & -0.8205346 & -0.8228615 & -0.8251884 & -0.8275153 & -0.9136098 & -0.9333883 \\
$\hat{\beta}_{4}$ & 0.8823875 & 0.8836893 & 0.8849912 & 0.8862930 & 0.8875949 & 0.9357634 & 0.9468291 \\
$Q B\left[\hat{\beta}_{G R}^{S}(d)\right]$ & 0.0609630 & 0.0599974 & 0.0590482 & 0.0581155 & 0.0571992 & 0.0348506 & 0.0328947 \\
$R\left[\hat{\beta}_{G R}^{S}(d), \beta\right]$ & 0.0519803 & 0.0512283 & 0.0504949 & 0.0497800 & 0.0490837 & 0.0363424 & 0.0369978 \\
$\Delta$ & -0.1498245 & -0.0142305 & -0.0134971 & -0.0127822 & -0.0120858 & 0.0006554 & 0.0000000 \\
$m s e(\hat{f})$ & 0.0791587 & 0.0790681 & 0.0811789 & 0.0850457 & 0.0871691 & 0.0800445 & 0.090521 \\
\hline \hline
\end{tabular}

The value of $\gamma$ represents the correlation between the two explanatory variables. Three different set of correlation corresponding to $\gamma=0.75, \gamma=0.85$ and $\gamma=0.95$ are considered. We selected a smooth function of the form

$$
f\left(t_{i}\right)=\frac{1}{10} \exp \left\{\sin \left(3 t_{i}\right) \cos \left(2 t_{i}\right)+\sqrt{t_{i}}\right\} \quad i=1, \ldots, n .
$$


Table 4. Evaluation of PRSGRLE at different $d$ values in model (20) with $\gamma=0.85$.

\begin{tabular}{cccccccc}
\hline$d$ & 0 & 0.2 & 0.4 & 0.6 & 0.8 & $d_{\text {opt }}=0.8585$ & 1 \\
\hline$\hat{\beta}_{1}$ & 1.0007353 & 1.0016658 & 1.0025964 & 1.0035271 & 1.0044576 & 1.0402857 & 1.0467998 \\
$\hat{\beta}_{2}$ & -0.0262929 & -0.0263761 & -0.0264592 & -0.0265424 & -0.0266255 & -0.0298262 & -0.0304082 \\
$\hat{\beta}_{3}$ & -0.8671964 & -0.8696624 & -0.8721285 & -0.8745946 & -0.8770606 & -0.9720041 & -0.9892665 \\
$\hat{\beta}_{4}$ & 0.8929779 & 0.8943340 & 0.8956900 & 0.8970461 & 0.8984021 & 0.9506097 & 0.9601020 \\
$Q B\left[\hat{\beta}_{G R}^{S}(d)\right]$ & 0.0297823 & 0.0288516 & 0.0279385 & 0.0270429 & 0.0261649 & 0.0057357 & 0.0048219 \\
$R\left[\hat{\beta}_{G R}^{S}(d), \beta\right]$ & 0.0484282 & 0.0476570 & 0.0469042 & 0.0461699 & 0.0454540 & 0.0319378 & 0.0324217 \\
$\Delta$ & -0.0160065 & -0.0152352 & -0.0144824 & -0.0137481 & -0.0130322 & 0.0004838 & 0.0000000 \\
$m s e(\hat{f})$ & 0.07806817 & 0.07901569 & 0.07938652 & 0.08096489 & 0.0816286 & 0.0851982 & 0.0870187 \\
\hline \hline
\end{tabular}

Table 5. Evaluation of SGRLE at different $d$ values in model (20) with $\gamma=0.95$.

\begin{tabular}{cccccccc}
\hline$d$ & 0 & 0.2 & 0.4 & 0.6 & 0.8 & $d_{\text {opt }}=0.8080$ & 1 \\
\hline$\hat{\beta}_{1}$ & 0.9485223 & 0.9504875 & 0.9524528 & 0.9544180 & 0.9563833 & 1.0271318 & 1.0458016 \\
$\hat{\beta}_{2}$ & 0.1703118 & 0.1678658 & 0.1654198 & 0.1629737 & 0.1605277 & 0.0724700 & 0.0492326 \\
$\hat{\beta}_{3}$ & -0.7060345 & -0.7104650 & -0.7148956 & -0.7193261 & -0.7237567 & -0.8832563 & -0.9253464 \\
$\hat{\beta}_{4}$ & 0.5877486 & 0.5926939 & 0.5976392 & 0.6025846 & 0.6075299 & 0.7855610 & 0.8325414 \\
$Q B\left[\hat{\beta}_{G R}^{S}(d)\right]$ & 0.2880230 & 0.2803591 & 0.272803 & 0.2653550 & 0.2580147 & 0.0656012 & 0.0381371 \\
$R\left[\hat{\beta}_{G R}^{S}(d), \beta\right]$ & 0.2426191 & 0.2360923 & 0.2297300 & 0.2235323 & 0.2174992 & 0.1099363 & 0.1171272 \\
$\Delta$ & -0.1254919 & -0.1189651 & -0.1126028 & -0.1064051 & -0.1003720 & 0.0071908 & 0.0000000 \\
$m s e(f)$ & 0.0388354 & 0.0388159 & 0.0387119 & 0.0387084 & 0.0386369 & 0.0386551 & 0.03811980 \\
\hline \hline
\end{tabular}

Table 6. Evaluation of PRSGRLE at different $d$ values in model (20) with $\gamma=0.95$.

\begin{tabular}{cccccccc}
\hline$d$ & 0 & 0.2 & 0.4 & 0.6 & 0.8 & $d_{\text {opt }}=0.8181$ & 1 \\
\hline$\hat{\beta}_{1}$ & 0.9318869 & 0.9334301 & 0.9349732 & 0.9365163 & 0.9380594 & 0.99438310 & 1.0082711 \\
$\hat{\beta}_{2}$ & 0.1334654 & 0.1305617 & 0.1276579 & 0.1247542 & 0.1218505 & 0.01586387 & -0.0102698 \\
$\hat{\beta}_{3}$ & -0.7402912 & -0.7451978 & -0.7501045 & -0.7550112 & -0.7599179 & -0.93901228 & -0.9831725 \\
$\hat{\beta}_{4}$ & 0.6711018 & 0.6773463 & 0.6835908 & 0.6898353 & 0.6960798 & 0.92400340 & 0.9802037 \\
$Q B\left[\hat{\beta}_{G R}^{S}(d)\right]$ & 0.1980750 & 0.1905074 & 0.1830875 & 0.1758154 & 0.1686910 & 0.0097781 & 0.0008489 \\
$R\left[\hat{\beta}_{G R}^{S}(d), \beta\right]$ & 0.2395765 & 0.2330098 & 0.2266074 & 0.2203691 & 0.2142951 & 0.1049730 & 0.1116387 \\
$\Delta$ & -0.1279377 & -0.1213711 & -0.1149686 & -0.1087303 & -0.1026563 & 0.0066657 & 0.0000000 \\
$m s e(\hat{f})$ & 0.0393271 & 0.0387613 & 0.0386098 & 0.0371127 & 0.0375382 & 0.0364906 & 0.0365120 \\
\hline \hline
\end{tabular}

for the whole simulation experiment. Using (18) and (19), then $n=50$ observations for the dependent variable are determined by

$$
y_{i}=\sum_{j=1}^{4} x_{i j} \beta_{j}+f\left(t_{i}\right)+\epsilon_{i}, \quad t_{i} \stackrel{i . i . d}{\sim} u(0,1), \quad i=1, \ldots, n .
$$

The parameters $\beta, H$ matrix and $h$ vector in (1.3) are chosen as the following forms respectively

$$
\beta=\left[\begin{array}{llll}
1 & 0 & -1 & 1
\end{array}\right]^{\prime} \quad H=\left(\begin{array}{cccc}
1 & -1 & 3 & 1 \\
3 & 2 & 1 & 0 \\
4 & -2 & 0 & 5
\end{array}\right) \quad h=\left[\begin{array}{lll}
0 & 0 & 0
\end{array}\right]^{\prime} .
$$


We use a third-order differencing coefficients, $\wp_{0}=0.8582, \wp_{1}=-0.3832, \wp_{2}=-0.2809, \wp_{3}=-0.1942$, in which case $m=3$. We further suppose that $\epsilon \stackrel{i . i . d}{\sim} N\left(0, \sigma^{2} V\right)$ for which the elements of $V$ are $v_{i j}=\left(\frac{1}{n}\right)^{|i-j|}$ and $\sigma^{2}=4.00$. For estimating the nonparametric part of the model, $f(t)$, we use a local linear Gaussian kernel regression which the kernel is the standard normal density. We use direct plug-in methodology to select the bandwidth of a local linear Gaussian kernel regression estimate, as described by Ruppert et al [19]. All computations were conducted using the statistical package $\mathrm{R}$ and the experiment was done with $10^{5}$ iteration. The ratio of largest eigenvalue to smallest eigenvalue of design matrix in model (20) is approximately $\frac{\lambda_{4}}{\lambda_{1}}=672.11,1549.80$ and 14495.28 for $\gamma=0.75,0.85$ and 0.95 , respectively. In Tables $1-6$, we computed the proposed estimators, respectively. We numerically estimated the $Q B($.$) (quadratic bias), R(),. \Delta=R\left(\hat{\beta}_{G R}^{S o r S+} ; \beta\right)-R\left[\hat{\beta}_{G R(d)}^{S o r S+} ; \beta\right]$ and $m s e[f(t), f(t)]=\frac{1}{n} \sum_{i=1}^{n}\left(f\left(t_{i}\right)-\hat{f}\left(t_{i}\right)^{2}\right.$ for different values of $d$ and $\gamma$. We found the best values of $d\left(d_{\text {opt }}\right)$ by plotting $\Delta$ versus $d$ for each proposed estimators (Fig.1). In Fig.1, in the left part, the risk of SGRLE (solid line) and PRSGRLE (dash line) and in the right part, the $\Delta$ for SGRRE (solid line) and PRSGDRRE (dash line) versus Liu parameter $d$ are plotted for different values of $\gamma$. In the continuation, Fig.2 shows the fitted function by kernel smoothing after estimation of the linear part of the model by $\hat{\beta}_{G R}^{S}\left(d_{o p t}\right)$ and $\hat{\beta}_{G R}^{S+}\left(d_{o p t}\right)$, that is, $y-X \hat{\beta}_{G R}^{S o r S+}\left(d_{o p t}\right)$ , respectively, for $\gamma=0.80,0.90$ and 0.99 with $n=500$.

\section{Conclusion}

We have combined the idea of the preliminary test and Stein-rule estimators with the Liu [12] estimator in difference-based approach to obtain a better estimator for the regression parameters $\beta$ in a semiparametric linear model. To combat multicollinearity in data, we defined two main estimator, difference-based Stein-type generalized restricted Liu estimator (SGRLE) and difference-based positive-rule Stein type generalized restricted Liu estimator (PSGRLE) for estimating $\beta$, when it is suspected that $\beta$ may belong to a linear subspace defined by $H \beta=h$. The performance of the estimators is compared based on the quadratic risk functions under both null and alternative hypotheses, which specify certain restrictions on the regression parameters. Under some conditions we show that the SGRRE and PRSGRRE are superior to other proposed estimators in the semiparametric linear model. From our simulation it is found that the Liu parameter $d$ is an decreasing function of $\gamma$ for domination of $S G R L E$ over $S G R E$ in the sense of quadratic risk function and the PRSGRLE is better than SGRLE for all values of $k$ and $\gamma$ in the sense of risk function.

\section{Acknowledgements}

The authors thank two referees and an associate editor for helpful comments and suggestions on earlier drafts of the paper.

\section{REFERENCES}

1. F. Akdeniz, E. Akdeniz Duran, M. Roozbeh, M. Arashi, Efficiency of the generalized difference-based Liu estimators in semiparametric regression models with correlated errors, Journal of Statistical Computation and Simulation, 85(1), 147-165, 2015.

2. M. Arashi, Preliminary test and Stein estimators in simultaneous linear equations, J Linear Algebra Appl. 436, 5: 1195-1211, 2012.

3. M. Arashi, B.M. Golam Kibria, M. Norouzirad, S. Nadarajah, Improved preliminary test and Stein-rule Liu estimators for the ill-conditioned elliptical linear regression model, J. Multivariate Anal. 124, 53-74, 2014.

4. M. Arashi, M. Roozbeh, Some improved estimation strategies in high-dimensional semiparametric regression models with application to riboflavin production data, Stat. Paper, 1-20, 2016.

5. N. Benda, Pre-test estimation and design in the linear model, Statist. Plann. Inference, 52: (2), 225-240, (1996).

6. L.D.Brown, L.Wang, T.T.Cai, A difference-based approach to the semiparametric partial linear model, Electron.J.Stat.5, 619-64, 2011.

7. D.G. Gibbons, A, Simulation study of some ridge estimators, J. Amer. Statist. Assoc. 76, 131C139, 1981

8. W. H?rdle, H. Liang, J. Gao,, Partially Linear Models, Physika Verlag, Heidelberg, 2000.

9. A.E. Hoerl, R.W. Kennard, Ridge regression: biased estimation in non-orthogonal problems, Technometrics, 12: (3), 55-67,1970.

10. G.G. Judge, M.E. Bock, The Statistical Implications of Pre-test and Stein-rule Estimators in Econometrics, North-Holland Publishing Company, Amsterdam, 1978.

11. K. Liu, A new class of biased estimate in linear regression, Comm.Statist.Theory Methods, 22:2, 393-402, 1993.

12. K. Liu, Using Liu-type estimator to combat collinearity, Comm. Statist. Theory Methods, 32: (5),1009-1020, 2003. 
13. M. Norouzirad, M. Arashi, Preliminary test and Stein-type shrinkage ridge estimators in robust regression, Stat. Paper, 1-34, 2017.

14. G.C. McDonald, D.I. Galarneau, A Monte Carlo evaluation of some ridge-type estimators, J. Amer. Statist. Assoc. 70, 407-416, 1975.

15. M.Muller, Semiparametric Extensions to Generalized Linear Models, Habilitationsschrift,2000.

16. C.R, Rao, H.Toutenburg, Shalabh, C.Heumann, Linear Models:Least Squares and Alternatives, Springer, Berlin, 2008.

17. M. Roozbeh, Shrinkage ridge estimators in semiparametric regression models, J.Multivariate Anal.136, 56-74, 2015.

18. M. Roozbeh, M. Arashi, Feasible ridge estimator in partially linear models, Journal of Multivariate, 116, 35-44, 2013.

19. D. Ruppert, SJ. Sheather, MP. Wand, An effective bandwidth selector for local least squares regression, Journal of the American Statistical Association 90: 432, 1257-1270, 1995.

20. A.K.Md.E. Saleh, Theory of Preliminary Test and Stein-type Estimation with Applications, John Wiley, New York, 2006.

21. A.K.Md.E. Saleh, B.M.G. Kibria, Performances of some new preliminary test ridge regression estimators and their properties, Comm. Statist. Theory Methods, 22: (10), 2747-2764, 1993.

22. C. Stein, Inadmissibility of the usual estimator for the mean of a multivariate normal distribution, in: Proceedings of the Third Berkeley Symposium, vol. 1, pp. 197C206, 1956.

23. P.A.V.B. Swamy, J.S. Mehta, A note on minimum average risk estimator for coefficients in linear models, Comm. Statist. Theory Methods, 6,1161-1186, 1977.

24. P.A.V.B. Swamy, J.S. Mehta, P.N. Rappoport, Two methods of evaluating Hoerl and Kennards ridge regression, Comm. Statist. Theory Methods, 6,1133-1155, 1978.

25. J.B. Wu, Y. Asr, A weighted stochastic restricted ridge estimator in partially linear model, Communications in Statistics-Theory and Methods, 46(18), 9274-9283, 2017.

26. A.Yatchew, Semiparametric Regression for the Applied Econometrican, Cambridge University Press,Cambridge, 2003.

27. B. Yuzbasi, S.E. Ahmed, D. Aydin, Ridge-type pretest and shrinkage estimations in partially linear models, Statistical papers. https://doi.org/10.1007/s00362-017-0967-8,2017.

28. B. Yuzbasi, Y. Asar, S.M. S?k, A. Demiralp, Improving estimations in quantile regression model with autoregressive errors, Thermal Science, https://doi.org/10.2298/TSCI170612275Y, 2018.

29. J. Li, W. Zhang, Z. Wu, Optimal zone for bandwidth selection in semiparametric models, J. Nonparametr.Stat. 23, 701-717, 2011.

30. Z. Zhong, Hu Yang, Ridge estimation to the restricted linear model, Comm. Statist. Theory Methods, 36:11, 2099-2115, (2007). 